\title{
The Contribution of Fibrinogen to Inflammation and Neuronal Density in Human Traumatic Brain Injury
}

\author{
Jenkins DR ${ }^{1}$, Craner MJ ${ }^{1}$, Esiri MM ${ }^{1}$, DeLuca GC $^{1}$ \\ ${ }^{1}$ Nuffield Department of Clinical Neurosciences, University of Oxford, Oxford, UK \\ Corresponding author: \\ Dr Damian Robert Jenkins MA BM BCh MRCP \\ damian.jenkins@ndcn.ox.ac.uk \\ Neuropathology, Nuffield Department of Clinical Neurosciences, Level 1 West Wing, John \\ Radcliffe Hospital, Oxford OX3 9DU. Tel +44 1865 274900. Fax +44 1865274950.
}

Additional authors:

Dr Matthew J Craner MB ChB PhD FRCP

matthew.craner@ndcn.ox.ac.uk

Muliple Sclerosis Clinical Trials Unit, Nuffield Department of Clinical Neurosciences, Level 3 West Wing, John Radcliffe Hospital, Oxford OX3 9DU. Tel +44 1865 231869. Fax +44 1865 231870.

Professor Margaret M Esiri DM FRCPath

margaret.esiri@ndcn.ox.ac.uk 
Page 2 of 58

2

Neuropathology, Nuffield Department of Clinical Neurosciences, Level 1 West Wing, John Radcliffe Hospital, Oxford OX3 9DU. Tel +44 1865 274900. Fax +44 1865274950.

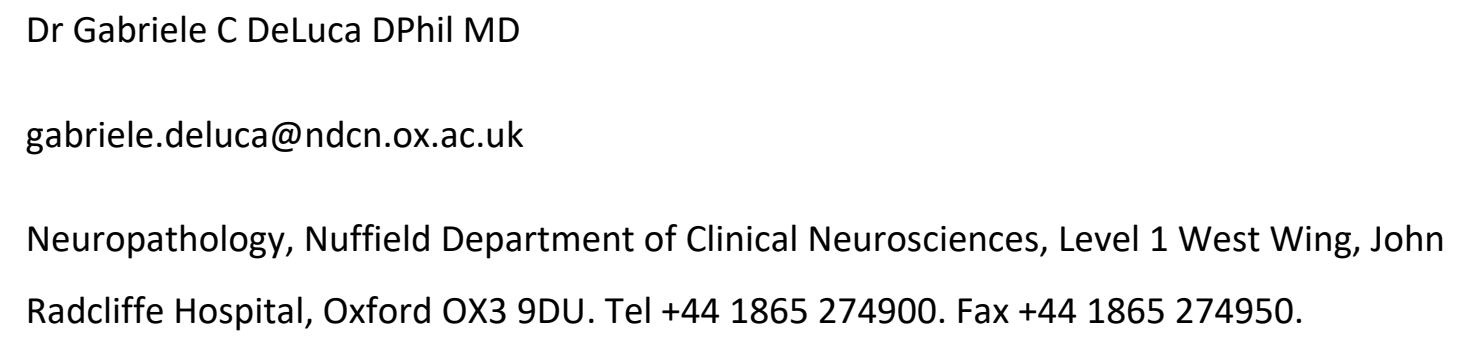




\section{Abstract}

Traumatic brain injury is a leading cause of death and disability, particularly among the young. Despite this, no disease-specific treatments exist. Recently, descriptions of BBB disruption and parenchymal fibrinogen deposition have been reported in acute traumatic brain injury and in long-term survival; however, their contribution to the neuropathology of TBI remains unknown. ${ }^{1,2}$ The presence of fibrinogen - a well-documented activator of microglia/macrophages - may be associated with neuroinflammation, and neuronal/axonal injury. To test this hypothesis, cases of human TBI with survival times ranging from 12 hours to 13 years (survival $<2$ months $n=15$, survival $>1$ year $n=6$ ) were compared with uninjured controls $(n=15)$. Tissue was selected from the frontal lobe, temporal lobe, corpus callosum, cingulate gyrus and brainstem, and the extent of plasma protein (fibrinogen and IgG) deposition, microglial/macrophage activation (CD68 and Iba-1 immunoreactivity), neuronal density, and axonal transport impairment ( $\beta A P P$ immunoreactivity) were assessed. Quantitative analysis revealed a significant increase in parenchymal fibrinogen and IgG deposition following acute TBI compared with long-term survival and control. Fibrinogen, but not IgG, was associated with microglial/macrophage activation and a significant reduction in neuronal density. Perivascular fibrinogen deposition was also associated with microglial/macrophage clustering and accrual of $\beta A P P$ in axonal spheroids, albeit rarely. These findings mandate the future exploration of causal relationships between fibrinogen deposition, microglia/macrophage activation and potential neuronal loss in acute TBI.

\section{Keywords}

TBI; fibrinogen; microglia; neurodegeneration. 


\section{Introduction}

Traumatic brain injury (TBI) accounts for $\approx 260$ hospitalizations or deaths per 100,000 people per year in Europe. ${ }^{3}$ Despite being common, it has no disease-specific therapies, and treatment is currently limited to supportive care. ${ }^{4}$ Secondary pathological processes as diverse as inflammation, dysregulated calcium signaling, and the toxic release of excitatory neurotransmitters have been targeted in clinical trials aimed at improving outcomes. However, none has conferred a survival benefit, or reduced morbidity, following TBI. ${ }^{567}$ This failure may partly be explained by a reliance on animal tissue for the identification of treatment targets. Experimental models inflict uniform patterns of injury and thus necessarily fail to mirror the complexity of human TBI. Trials are further impeded by a lack of validated imaging and other biomarkers permitting patients to be grouped and treated according to shared pathophysiological features. Given these limitations, there is an urgent need to explore the processes underpinning TBI in human tissue: a need that can be met by high quality neuropathological studies.

To develop new treatment targets requires a better understanding of the mechanisms underpinning secondary cell death in TBI, and a key therapeutic candidate is the BBB. It has been shown that TBI induces a breakdown in the BBB, starting within minutes of injury and lasting for days. ${ }^{1,8}$ The effect may be permanent, with long-term head injury survivors having evidence of ongoing BBB disruption for months or years after impact. ${ }^{9}$ The effects of BBB damage are varied but include oedema and inflammation, which likely contribute to cell death. ${ }^{10}$ What is more, BBB disruption can permit the extravasation of plasma proteins into the brain parenchyma, as has recently been shown in human tissue. ${ }^{2}$ One such protein - fibrinogen, as well as its breakdown product fibrin (herein jointly referred to as fibrinogen) - is capable of binding to, and activating,

monocyte-derived cells, provoking and sustaining an inflammatory response. ${ }^{11}$ This has been demonstrated mostly clearly in the experimental autoimmune encephalomyelitis (EAE) model of MS. Here, the breakdown of the BBB leads to fibrinogen deposition, which 
promotes perivascular clustering of microglia/macrophages, with subsequent axonal transection. ${ }^{12}$ A pathological role for BBB disruption and fibrinogen deposition is emerging in other neurological conditions, including $A D$, epilepsy, and peripheral nerve injury. ${ }^{13,14}$ 15, 16 Experimentally preventing the interaction between fibrinogen and microglia/macrophages not only dampens down inflammation, it also decreases the frequency with which pathological markers are observed in both $A D$ and MS. ${ }^{17,}{ }^{18}$ In addition to its role in disease, parenchymal fibrinogen deposition is self-propagating, as its presence alters the expression of endothelial cell tight junction proteins, leading to an increase in vessel permeability. ${ }^{19,20}$ This may be pertinent to TBI in which persistent protein extravasation and neuroinflammation might contribute to the development of post-traumatic epilepsy (PTE). ${ }^{21}$

Based on these observations, we hypothesized that the parenchymal deposition of fibrinogen would be associated with both microglial/macrophage activation and neuronal/axonal injury in TBI. Using human post-mortem tissue, we demonstrate that fibrinogen and IgG are acutely deposited in multiple brain regions following trauma, in patterns not previously described. The presence of fibrinogen in acute TBI, but not in cases of long-term survival, is associated with microglial/macrophage activation in both the grey matter (GM) and white (WM), which is further associated with a reduction in neuronal density, and, in rare instances, with $\beta A P P$ accrual in axonal spheroids. No relationship is seen between IgG and inflammation, reduced neuronal density or $\beta A P P$ accrual in axons. These findings highlight a putative contribution of fibrinogen to inflammation and neuronal in acute human TBI.

\section{Materials and Methods}

Human post-mortem tissue was obtained from the Oxford Brain Bank having been collected in accordance with the UK Human Tissue Act, with informed consent provided 
6

for all donations (Oxford Brain Bank Research Ethics Committee approval number 07/0606/85). Three cohorts were created: acute TBI (survival <2 months); long-term survival (survival > 1 year); and control. All cases of TBI were moderate-severe according to the Mayo criteria (worst GCS in first 24 hours <13, and/or presence of hematoma, contusion, subarachnoid hemorrhage). ${ }^{6}$ TBI severity was further subdivided according to the admission GCS (severe injury $\leq 8$, moderate injury 9-12). ${ }^{7}$ Where GCS was not available, cases were subdivided according to available information, with coma taken to represent a GCS <8 (severe), and focal lesions with no mention of coma/reduced GCS taken to represent moderate TBI. The control cohort consisted of age/sex-matched cases with no known history of TBI and without clinical evidence of dementia (Table 1).

Whole brains were fixed by immersion in $10 \%$ formalin solution for $\geq 4$ weeks and $\leq 4$ months. Cases with longer fixation times were omitted to ensure the preservation of antigenicity for immunohistochemistry (IHC). ${ }^{24}$ Where available, tissue blocks were acquired in each case from the frontal lobe, anterior corpus callosum (with accompanying cingulate gyrus), temporal lobe, thalamus (including neighbouring internal capsule), and brainstem (pons). The chosen regions are vulnerable to TBI and have been reported to undergo plasma protein deposition following trauma. ${ }^{25}$

\section{Immunohistochemistry, immunofluorescence and image acquisition}

Staining was performed on sections of $6 \mu \mathrm{m}$ thickness. Tissue was deparaffinised and rehydrated. For IHC, endogenous peroxidase activity was quenched by exposure to $3 \%$ $\mathrm{H}_{2} \mathrm{O}_{2}$ for 30 minutes. Specific antigen retrieval methods, primary antibody concentrations, and incubation times are provided (Supplementary Figure 1A). Following incubation with a suitable secondary antibody, IHC results were visualized using a 3,3'-diaminobenzidine (DAB) EnVision+ kit (Dako, Glostrup, Denmark), prior to counterstaining with hematoxylin. For immunofluorescence, results were visualized with Alexa Fluor ${ }^{\circledR}$ secondary antibodies, 
followed by exposure to Sudan black for 10 minutes, to reduce autofluorescence (Supplementary Figure 1B). The specificity of fibrinogen staining was confirmed by two means. First, staining was performed on multiple sclerosis tissue with well documented fibrinogen deposition, and was seen in the same distribution as that reported. ${ }^{26}$ Second, staining was performed with both monoclonal and polyclonal fibrinogen antibodies, developed by separate companies, and the results compared. No significant differences in staining were noted (Figure 1). Negative controls were created by omitting the primary antibody. Where available, tissue sections stained with haematoxylin and eosin (H\&E) were acquired for each case from their original post-mortem study. Whole slide images were digitally captured by an Aperio Scanscope ${ }^{\circledR}$ AT slide scanner (Leica Microsystems, Wetzlar, Germany; images viewable at x1 - x400 magnification) or Olympus Fluoview FV1000 (confocal).

\section{Analysis}

Associations between plasma protein deposition, inflammation, and markers of neuronal/axonal injury were tested:

1. Regionally (on the entire GM and entire WM compartments of each cortical tissue section, in the WM of the corpus callosum and internal capsule, in the GM of the thalamus, and in a whole hemi-pons);

2. In trajectories emanating away from areas of dense fibrinogen deposition, towards areas of low or absent fibrinogen deposition.

Sections were analysed blind to cohort.

$\underline{\text { Regional Analysis - Semi-quantitative }}$

A semi-quantitative analysis of $\mathrm{CD} 68$, Iba-1, and $\beta A P P$ staining was performed according to previously published methods. ${ }^{27}$ Briefly: 
1. Tissue sections stained for CD68 and lba-1 were scored according to the predominant microglial/macrophage morphology, i.e. predominantly ramified (0), mixed (1), or predominantly amoeboid (2). For the purposes of this study, an amoeboid morphology was ascribed to microglia/macrophages with rounded cell bodies, devoid of processes. Separate scores were generated for the GM and WM.

2. Axonal pathology was scored in the WM alone, and was identified by the presence of $\beta$ APP-positive axonal bulbs/varicosities. The number of axonal bulbs/varicosities was scored semi-quantitatively as being absent/minimal ( $<10$ bulbs or varicosities per section) (0), moderate (1), or extensive (2). \{Johnson:2013jg\} Within each tissue section, axons demonstrating evidence of hypoxic-ischaemic injury (HII) were not included in the score. HII was identified by the presence of $\beta$ APP-positive axons in a geographical pattern, scattered in WM rather than oriented along tracts, lacking longitudinal profiles; ${ }^{28} 29$

3. Fibrinogen and IgG deposition were also scored using a previously employed method. ${ }^{2}$ Plasma proteins were scored as absent (0), sparse (1), moderate (2), or extensive (3).

\section{Regional Analysis - Quantitative}

Automated quantification of fibrinogen, IgG, $\beta A P P, C D 68$ and Iba-1 staining was performed on tissue sections from each available brain region, with separate counts made in the GM and WM. Quantification was performed using the Aperio ImageScope Positive Pixel Count tool (Leica Biosystems, Wetzlar, Germany), which measures the number of positively and negatively stained pixels to generate a value for positivity (positivity = total number of positive pixels $\div$ total number of pixels). A DAB-stained image was used to threshold the algorithm, ensuring all positively stained pixels were identified and counted. A section of control tissue, stained only with haematoxylin, was used to confirm the algorithm specificity (Supplementary Figure 1C). Therein: 
- Luminal fibrinogen intraneuronal fibrinogen, was excluded from analysis through the application of an occlusion tool;

- Impaired axonal transport, resulting in $\beta A P P$ staining (a surrogate marker of axonal injury) was quantified in the WM. Axons demonstrating evidence of hypoxicischaemic injury (HII) were masked by an occlusion tool, ensuring their exclusion from analysis;

- Total microglial/macrophage burden, and the burden of activated/lysosomal microglia/macrophages, was measured by quantifying Iba-1 and CD68 staining respectively. ${ }^{30}$ The abundance of activated/lysosomal microglia/macrophages relative to the total microglial/macrophage burden was assessed by means of a CD68/Iba-1 ratio.

Neuronal counts were performed on tissue sections stained for NeuN, with counting frames created at $\times 200$ magnification. NeuN-positive cells were counted if they contained both a nucleus and a single, large nucleolus. Therein:

- Cortical neuronal counts were made on sections of frontal lobe, temporal lobe, and cingulate gyrus. Ten fields of view (FOV) were created in each section: five each in the supragranular (SG) and infragranular (IG) layers (layers I-III and IV-VI respectively);

- In the brainstem, up to twelve FOV were created along the midline of the hemipons, between the dorsal and ventral surfaces;

- In the thalamic grey matter, ten FOV were created between the ventricular surface, medially, and the internal capsule, laterally;

Neuronal density was calculated by dividing the number of cells by the area of the counting frame. To correct for oedema, the area within each frame occupied by extracellular space (identified by the algorithm as white space) was measured and subtracted from the area of the counting frame. ${ }^{31}$ Neuronal counts in each tissue section 
10

were averaged. In a subset of images, counts were verified by a second assessor with good inter-observer agreement $\left(r_{s} 0.989, R^{2} 0.978\right)$.

Loss of NeuN antigenicity has been noted through mechanisms other than cell loss, especially due to neuronal ischemia. Failing to include such neurons in cell counts can result in inaccurate estimates of neuronal density. ${ }^{32} 33$ The validity of data generated from NeuN-stained sections was validated by comparing neuronal counts on H\&E-stained sections. In H\&E sections, neurons were identified by the presence of intact cell and neuronal membranes, and the presence of a single, large nucleolus. Neuronal density measured by NeuN and by H\&E were compared, showing strong agreement between the two methods $\left(r_{s} 0.88, P<0.0001\right)$.

The thickness of the corpus callosum and cortex were measured. To calculate corpus callosum thickness, measurements were taken of the superior-to-inferior extent in the midline, at the juncture with the cingulate gyrus, and at the midway position between these points. The three figures were averaged. In tissue sections containing cortex, cortical thickness was measured at either end of the cortical ribbon in each section, and at the midway point. The three figures were averaged.

Findings from the above were used to assess for associations between plasma protein deposition, inflammation, and neuronal density in the $G M$, and between plasma protein deposition, inflammation and axonal injury in the WM of each brain region. 
Trajectory Analysis

\begin{abstract}
Next, we performed a trajectory analysis. This permitted us to focus on areas of pathology identified by plasma protein deposition. Where present, gradients of fibrinogen and IgG were identified in each tissue section. Three FOV were created along the plasma protein gradient: Area 1 was focused on the area of greatest protein deposition; Area 3 at the area with least/no protein deposition; with Area 2 equidistant between the two. Areas 1-3 were overlaid onto sequentially cut sections stained for NeuN, $\beta A P P, C D 68$ and Iba-1. Gradients were analysed separately in the GM and WM. When assessing the GM, care was taken to maintain the trajectory within the same cortical layer (i.e. supragranular/infragranular), and to avoid regions with clear evidence of NeuN loss but preserved neuronal morphology (taken as a marker of potential ischemic neuronal injury). In the WM, areas that demonstrated axonal HII were excluded. Neuronal density, $\beta$ APP accrual, and CD68/Iba-1 were quantified as before, and areas along the protein gradient were compared with each another (Area1:Area2 and Area1:Area3).
\end{abstract}

\title{
Statistical analysis
}

Data were analysed by one-way ANOVA testing and significance confirmed using Tukey's honest significant difference (HSD) test. Results were considered positive if the two-tailed $P<0.05$. Figures contain Tukey HSD results and standard deviations (SD). Correlations between variables were testing us the Spearman's Rank correlation coefficient, and results considered significant where $P<0.05$. Analysis/graphical output were performed in GraphPad Prism 7, and graphs include error bars showing SD. Graphs comparing plasma protein deposition between cohorts represent the combined data from all brain regions. Correlation graphs comparing fibrinogen or IgG, CD68/Iba-1 and neuronal density use data combined from all brain regions except the brainstem, where it was known that no relationship existed. Regression analysis was performed using SPSS v24 and 
results considered significant where $P<0.05$. No multicollinearity was found on testing (Variance Inflation Factor $<5$ in each instance).

RESULTS

Cohort

We identified cases of acute TBI $(n=15)$, long-term survival $(n=6)$ and control $(n=$ 15) for inclusion in the study, resulting in a total of 217 tissue blocks from seven brain regions (Table 1). Contusion material was present in a small number $(n=3)$ of the tissue sections evaluated, and was limited to the WM (Table 1, Figure 1). Cases were matched for age $(P=0.68)$ and sex $(P=0.50)$, and did not differ in respect of post-mortem (PM) interval $(P=0.95)$. The cohorts differed in terms of time to death and cause of death $(P<0.0001)$, and $4 / 6$ cases (67\%) of long-term survival suffered PTE.

\section{Fibrinogen Deposition}

Parenchymal fibrinogen deposition was greater in acute TBI than in long-term survival or control. Staining appeared multifocal, concentrated around blood vessels in both the GM and WM. In many cases, staining was both diffuse and confluent, but retained evidence of perivascular concentration. The same pattern emerged regardless of antibody used (monoclonal vs polyclonal; Figures 1-2). Fibrinogen was also seen in all tissue sections containing contusion: both in, and around, the lesion. In addition, intraneuronal fibrinogen was observed in approximately one quarter of tissue sections ( $\mathrm{n}=$ $35 / 151)$. The deposition of fibrinogen showed regional variation, being most abundant in tissue sections from the thalamus and least abundant in sections of brainstem and internal 
capsule (Supplementary Figure 3). The deposition of fibrinogen in each case is provided at Supplementary Figure 2.

Fibrinogen deposition was rare in cases of long-term survival and control. Where seen, fibrinogen was seen in the perivascular space, with some limited extension into the WM (Figure 2D-E). Of interest, the $4 / 4$ cases of long-term survival, and 4/12 control cases exhibiting fibrinogen deposition, also had a history of PTE or died from complications of epilepsy.

Semi-quantitative scoring confirmed that fibrinogen deposition was greater in acute TBI than in long-term survival or control. This was true of both the GM (Mean score $=1.78$ vs 0.26 vs $0.41, \mathrm{P}<0.0001$ ) and $\mathrm{WM}$ (Mean score $=1.42$ vs 0.23 vs $0.33, \mathrm{P}<0.0001$ ) Quantitative results revealed a similar picture, with significantly greater fibrinogen deposition in acute TBI (Mean GM positivity $=0.20$ vs 0.05 vs $0.04, P<0.0001$; Mean WM positivity $=0.16$ vs 0.05 vs $0.03, P<0.0001$ ) (Figure 1 ). This finding held true when tissue sections containing contusion were omitted from analysis. In acute TBI, there was no significant difference between GM and WM fibrinogen deposition (P 0.43), but regional variation in deposition was noted (Supplementary Figure 3). Tissue sections were dichotomized to those containing intraneuronal fibrinogen and those without, and the mean GM fibrinogen was compared between groups. This demonstrated that sections with positive intraneuronal staining had greater mean GM fibrinogen deposition that sections without neuronal staining (Mean GM positivity $=0.347$ vs $0.08, \mathrm{P}<0.0001$ ). Finally, semi-quantitative and quantitative fibrinogen measurements were correlated $(0.67, \mathrm{P}$ $<0.0001$ ), and no association was seen between PM interval and fibrinogen deposition ( $P$ $0.12)$. 


\section{IgG Deposition}

Greater IgG staining was seen in acute TBI compared to long-term survival and control (Figure 1E). Like fibrinogen, IgG staining appeared concentrated around blood vessels in both the GM and WM, with disuse, confluent staining also seen. A further breakdown of IgG deposition by case and by brain region is included in Supplementary Figure 2. All cases of fibrinogen deposition had concomitant IgG staining, as seen in tissue samples containing contusions and hematomata (Figure 1). However, parenchymal IgG deposition was also seen alone, especially in the brainstem. What is more, within a given tissue section, it was possible to see fibrinogen in the GM and WM, and IgG in the WM alone (Figure 1A-C).

Semi-quantitative analysis revealed significantly greater IgG staining in acute TBI than in long-term survival or control (Figure 1E), and this was true in both the GM (Mean score $=1.95$ vs 0.83 vs $0.83, \mathrm{P}<0.0001$ ) and $\mathrm{WM}$ (Mean score $=1.53$ vs 0.67 vs $0.54, \mathrm{P}$ $<0.0001)$. This finding was confirmed by quantitative analysis: Mean GM positivity $=0.32$ vs 0.09 vs $0.05, \mathrm{P}<0.0001$; Mean $\mathrm{WM}$ positivity $=0.24$ vs 0.10 vs $0.07, \mathrm{P}<0.0001$. The significance of IgG deposition in acute TBI was not altered by the omission of tissue sections containing contusion, and IgG deposition in cases of long-term survival or control was not significant. Furthermore, there was no significant difference in IgG deposition between brain regions ( $P$ 0.95). Compared to fibrinogen, there was greater IgG than fibrinogen staining in the GM (Mean GM IgG vs Mean GM fibrinogen positivity $=0.32$ vs 0.20, $\mathrm{P}$ 0.002) and a trend towards greater $\lg G$ than fibrinogen deposition in the WM (Mean WM positivity $=0.24 \vee 0.16, \mathrm{P} 0.058$ ). Semi-quantitative and quantitative measures of IgG were strongly correlated $\left(r_{s} 0.84, P<0.0001\right)$, and IgG staining did not correlate with PM interval (P 0.81). 
Inflammation

Acute TBI was associated with a microgliosis, with amoeboid microglia/macrophages seen across brain regions, both with and without contusion. Morphological change to an amoeboid cell shape was best seen with CD68 staining. Amoeboid microglial/macrophages were also seen in long-term survival and control, but a predominance of amoeboid over ramified cells was seen only in the WM of the corpus callosum, internal capsule, and frontal lobe in cases of long-term survival. In a small number of cases of acute TBI $(n=5 / 15)$ and long-term survival $(n=2 / 6)$, microglia/macrophages were observed in clusters around blood vessels. These vessels had in common a pattern of dense, perivascular fibrinogen deposition (Supplementary Figure 5). Where present, clustering was limited to one or two vessels per section, and was, therefore, a rare finding. Compared to vessels within the same tissue section but without perivascular fibrinogen, affected blood vessels had a greater density of amoeboid microglia/macrophages in a cuff around the vessel periphery. There was no evidence of microglial/macrophage clustering around of blood vessels with perivascular IgG deposition alone. Amoeboid microglia were also seen at the edges of contusions where they overlapped with fibrinogen and IgG staining.

A semi-quantitative analysis of CD68-stained tissue sections in acute TBI confirmed a change from a ramified microglial/macrophage morphology to one in which amoeboid cells were more prevalent. This change was greater than that seen long-term survival and control in the GM (Mean GM CD68 score $=1.28$ vs 1.02 vs $0.65, \mathrm{P}<0.0001$ ) and WM (Mean WM CD68 score $=1.03$ vs 0.87 v 0.20, P <0.0001). Interestingly, the Mean WM CD68 score was also significantly greater in long-term survival than in control (P 0.0004). There was no difference in CD68 score between brain regions, and the difference between GM and WM CD68 was not significant. 
Iba-1-stained tissue sections showed a similar shift in morphology from ramified to amoeboid cells in acute TBI (Mean GM Iba- 1 score $=0.46$ vs 0.21 vs $0.10, P<0.0001$; Mean WM Iba-1 score $=0.82$ vs 0.36 vs $0.25, P<0.0001$ ). The difference was subtler with Iba- 1 staining than CD68, insofar as a smaller proportion of cells appeared amoeboid. No difference emerged between long-term survival and control, and there was no variation in Iba-1 score by brain region. Whilst scores of CD68 and Iba-1 were significantly correlated $\left(r_{s} 0.54, P<0.0001\right)$, the GM CD68 score was significantly greater than the GM Iba-1 score in acute TBI (Mean score $=1.28$ vs $0.46, \mathrm{P} 0.0002)$.

Quantitatively, acute TBI was associated with a significant increase in CD68 staining compared with long-term survival and control in both the GM and WM (Mean GM CD68 positivity $=0.025$ vs 0.015 vs 0.015 , $\mathrm{P} 0.0002$; Mean WM CD68 positivity $=0.032$ vs 0.030 vs $0.021 \mathrm{P}$ 0.0003). A significant increase in CD68 staining was also noted when comparing corpus callosum sections from cases of long-term survival and control (Mean WM CD68 positivity $=0.038$ vs $0.023, \mathrm{P} 0.030$ ). By comparison, Iba-1 positivity was not significantly greater in acute TBI than in long-term survival or control in either the GM (Mean Iba-1 positivity $=0.062$ vs 0.061 vs 0.064 , $\mathrm{P}$ 0.22) or WM (Mean Iba-1 positivity = 0.065 vs 0.079 vs $0.077, P$ 0.22). Semi-quantitative measurements correlated with quantitative measurements of $\operatorname{CD68}\left(r_{s}\right.$ 0.36, $P$ 0.004) and lba-1 staining $\left(r_{s} 0.36, P\right.$ $<0.0001)$.

The CD68/Iba-1 ratio, comprising the quantitative measurements of CD68 and Iba1, was increased in acute TBI compared to long-term survival and control, thus demonstrating an increase in lysosomal and phagocytic activity relative to overall microglial/macrophage density. The increase in CD68/Iba-1 was seen in both the GM (Mean GM CD68/Iba-1 = 0.39 vs 0.24 vs 0.22, P 0.009) and WM (Mean WM CD68/Iba1 = 0.45 vs 0.46 vs $0.24, P$ 0.031), and was present across all brain regions except the brainstem. Compared to control, long-term survival was associated with an increase in CD68/Iba-1 in the corpus callosum (Mean WM CD68/Iba1 = 0.81 vs $0.21, \mathrm{P} 0.008$ ). 


\section{Neuronal density and axonal $\beta$ APP accrual}

In acute TBI, neuronal density appeared reduced, and the normal, cortical lamination was often disrupted. In cases with a reduced number of NeuN-stained cells, H\&E sections showed neurons with shrunken cytoplasm, and dark, sometimes pyknotic nuclei. Not all neurons that had lost NeuN staining appeared necrotic or damaged, retaining intact cell and nuclear membranes, and preserved cytoplasm volumes. These cells were thought to indicate ischemic neurons.

Quantitatively, neuronal density was reduced in acute TBI compared to long-term survival and control (Neurons $/ \mathrm{mm}^{2}=147$ vs 213 vs $218, \mathrm{P}<0.0001$ ). In acute TBI, there was no significant difference in neuronal density between the frontal lobe (186 neurons $/ \mathrm{mm}^{2}$, SD 67), cingulate gyrus (174 neurons/mm², SD 42), temporal lobe (167 neurons $/ \mathrm{mm}^{2}$, SD $65)$, or brainstem (110 neurons $/ \mathrm{mm}^{2}, \mathrm{SD} 46$ ); and neuronal density was similar in the SG and IG layers (Neurons/mm2 $=181$ vs 169, P 0.47). The neuronal density of the thalamus was lower than that of cortical brain regions in acute TBI, but this was equally true of control tissue (Thalamic neurons $/ \mathrm{mm}^{2}$ acute TBI = 61, SD 65, P 0.0003; control = 99, SD 35, $\mathrm{P}<0.0001)$.

Compared with control, acute TBI was not associated with a significant difference in cortical thickness. However, a significant reduction in thickness was seen with long-term survival compared with control (acute TBI vs long-term survival vs control thickness $/ \mathrm{mm}=$ 2.72 vs 2.44 vs 2.92 , P 0.005$)$. Comparing cohorts also revealed a significant reduction in corpus callosum thickness in long-term survival (acute TBI vs long-term survival vs control thickness $/ \mathrm{mm}=52.6$ vs 38.9 vs $50.5, \mathrm{P} 0.024)$. 
WM $\beta A P P$ staining was measured semi-quantitatively and quantitatively. Both showed a significant increase in WM $\beta$ APP in acute TBI compared to long-term survival and control ( $\beta$ APP score $=1.0$ vs 0.1 vs $0.0, P<0.0001 ; \beta$ APP positivity $=0.05$ vs 0.00 vs $0.00, P$ $<0.0001)$. There was a strong correlation between quantitative and semi-quantitative scores $\left(r_{s}\right.$ 0.70, $\left.P<0.0001\right)$. Significant $(P$ 0.046) regional variation in $\beta A P P$ staining was noted, with greater staining in the internal capsule and corpus callosum (Positivity $=0.18$ and 0.11 respectively) compared to cortical WM and brainstem staining (e,g. brainstem $\beta A P P$ positivity $=0.02)$. Axonal accrual of $\beta$ APP was also seen scattered in the $W M$, outside of long WM tracts, especially around blood vessels. This was considered to represent HII. Little $\beta A P P$ staining was seen beyond two weeks.

\section{Relationships in acute TBI}

Automated pixel counts of plasma protein deposition, inflammation, and neuronal density/axonal $\beta$ APP accrual were used to test for associations between these factors. This revealed that, in acute $\mathrm{TBI}$, fibrinogen significantly and negatively correlated with neuronal density $\left(r_{s}-0.59, \mathrm{P}<0.0001\right.$; Figure $\left.3 \mathrm{~A}\right)$. The correlation strength varied by brain region, with no association seen between fibrinogen and neuronal density in the brainstem $\left(r_{s}\right.$ 0.08, P 0.82; Supplementary Figure 4). No association was seen between GM IgG deposition and neuronal density $\left(r_{s} 0.10, P\right.$ 0.43). Whilst axonal spheroid stained for $\beta A P P$ were rarely observed around fibrinogen-cuffed WM blood vessels, no correlation emerged between overall WM fibrinogen deposition and $\beta$ APP staining $\left(r_{s} 0.14, P\right.$ 0.37; Supplementary Figure 5). Similarly, WM IgG did not correlate with $\beta A P P\left(r_{s} 0.04, P 0.80\right)$.

Fibrinogen deposition in acute TBI correlated significantly with CD68/Iba-1 $\left(r_{s} 0.62\right.$, $P<0.0001$ ): an association seen in both the GM and WM. The strength of the association 
varied by brain region, but insignificantly so (P 0.46; Supplementary Figure 4), and no correlation was seen between fibrinogen and CD68/Iba-1 staining in the brainstem $\left(r_{s} 0.31\right.$, $P$ 0.32). No relationship emerged between WM CD68/Iba-1 and $\beta A P P$ in any brain region, and IgG deposition in acute TBI did not correlate with CD68/Iba-1, neuronal density, or axonal $\beta$ APP accrual.

A regression analysis, performed on the combined data of GM from all brain regions save the brainstem, which showed insignificant fibrinogen deposition compared with control, showed an interaction between GM fibrinogen, GM CD68/Iba-1, and neuronal density in acute TBI $\left(R^{2} 0.44, P<0.0001\right)$. Specifically, increasing fibrinogen deposition was associated with increasing CD68/Iba-1 and decreasing neuronal density. No interaction was seen between WM fibrinogen and $\beta$ APP or WM CD68/Iba-1 and $\beta A P P$. However, an association was noted between WM fibrinogen and WM CD68/Iba-1 $\left(R^{2} 0.26\right.$, P 0.045) (Supplementary Figure 5). Finally, there was no relationship between IgG deposition, microglial/macrophage activation and neuronal density/axonal injury in acute TBI.

The statistical relationships were further explored by assessing the state of microglial/macrophage activation, and the presence of neuronal/axonal markers, along fibrinogen and IgG gradients. Within individual tissue sections, we tested the spatial overlap of GM fibrinogen/IgG deposition, GM microglial/macrophage activation, and neuronal density. This revealed that acute fibrinogen deposition co-localised with areas of microglial/macrophage activation (an increased proportion of amoeboid cells, and an increase in CD68 staining), and areas of greatest reduction in neuronal density (Figure 3B). With increasing distance from the area of peak fibrinogen deposition, CD68/Iba-1 fell, and neuronal density increased. This was not the case for IgG gradients. A similar strategy was used to look at fibrinogen and IgG gradients in the WM, revealing that fibrinogen, but not IgG, overlapped with activated WM microglia/macrophages. However, neither fibrinogen nor IgG routinely overlapped with areas of peak $\beta A P P$ staining. 


\section{DISCUSSION}

\section{Acute TBI results in significant plasma protein deposition in the brain}

In this study, we found that the parenchymal deposition of fibrinogen and IgG was significantly increased following acute TBI. In contrast to the findings of others, we did not observe a significant increase in fibrinogen or $\lg G$ deposition with prolonged (>1 year) survival. ${ }^{2}$ Where seen, plasma protein deposition in long-term survival centred on perivascular space, with some extension into the surrounding WM. This was evident only in cases of PTE. This discrepancy may be one of tissue ascertainment, with potential differences in injury patterns, clinical care, or the incidence/severity of PTE compared with tissue from other studies. The latter is particularly important, as PTE is known to be independently associated with BBB disruption and plasma protein extravasation. Whilst out study suggests that fibrinogen deposition is a feature of PTE rather than long-term survival, no firm conclusions can be drawn from the limited number of cases in this study. However, our finding underscores the importance of including data on PTE in any future neuropathological TBI studies that include long-term survivors. 9, 15

Although IHC cannot determine the provenance of parenchymal IgG or fibrinogen, it is likely that both enter the CNS when the BBB is impaired, perhaps explaining the accentuation around blood vessels. ${ }^{34,} 35$ In our study, IgG deposition occurred in all brain regions and in most cases of acute $\mathrm{TBI}$, implying that some increase in BBB permeability occurs almost globally in head trauma. Significantly greater IgG than fibrinogen deposition was seen in the internal capsule, corpus callosum, and brainstem, whilst similar amounts of the two proteins accrued in other brain regions. It is not known whether an equal presence of $\operatorname{lgG}$ and fibrinogen serves as a metric of greater BBB damage than the presence of IgG alone: however, fibrinogen is a larger molecule than IgG (11.0nm vs $5.3 \mathrm{~nm}$ ) and is likely to require a greater increase in BBB permeability to gain entry to the 
CNS. Assuming the combined deposition of IgG and fibrinogen is indicative of greater BBB damage, our findings imply that the BBB is less severely affected in acute $T B I$ in the brainstem and large white matter tracts. Whilst this finding might simply reflect the pattern of injuries suffered by our cohort, it could also speak to differences in the anatomy of these regions, the orientation of their blood vessels, or in the local composition of the BBB, any of which might alter vessel integrity. For instance, the local arrangement of blood vessels relative to the parenchyma, or variations in the composition of the extracellular matrix, might allow vessels in such locations to withstand greater external forces than vessels in other brain regions. ${ }^{36}$

IgG and fibrinogen were observed in the corpus callosum in acute TBI, albeit to a lesser extent than in other brain regions. Whilst this is at odds with one previous histopathological report, our observation is consistent with imaging studies that demonstrate frequent WM microhaemorrhages following trauma, especially in the corpus callosum. ${ }^{2,37}$ Clearly, the presence of blood presupposes the presence of plasma proteins, and it is presumed that the discrepancy reflects differences in injury patterns between our respective cohorts. Of note, fibrinogen deposition was greatest in the thalamus. The thalamus has long been known to suffer significant injury following $\mathrm{TBI}$, incurring the greatest haemodynamic impairment of any brain region, and having significant neuronal loss across its various nuclei. ${ }^{38} 3940$ Furthermore, the thalamus is a site of significant inflammation following acute $\mathrm{TBI}$, and changes in its microstructural integrity correlate with cognitive impairment in long-term survivors. ${ }^{41}$ Our finding adds to a growing literature that suggests thalamic injury in head trauma is a significant in TBI, and may be an important determinant of outcomes.

Finally, we noted that individual plasma proteins were deposited in heterogeneous locations following injury. For example, whereas fibrinogen staining might be seen predominantly in the GM in a given tissue section, IgG staining might be discovered in the GM, GM and WM, or in the WM alone. This likely reflects the differing fates of fibrinogen 
and IgG once in the parenchyma: the former, which is part cleaved to an insoluble, fibrillar form (fibrin), can become enmeshed in the parenchyma; on the other hand, IgG, which is not cleaved and does not become fixed, might be expected to spread away from its point of entry into the CNS, passing preferentially out of the GM and through the more commodious paths found in the WM. ${ }^{42}$ Whilst it might be expected that fibrinogen, converted to fibrin, remains in the parenchyma longer than $\lg G$, we did not identify this in our tissue cohort. This differing fates will need to be examined in a larger cohort containing cases with various survival times.

\section{Fibrinogen deposition is associated with microglial/macrophage activation and a reduction in neuronal density}

Next, we found that fibrinogen deposition was associated with microglial/macrophage activation. Cells proximate to fibrinogen had retracted processes and a rounded, amoeboid morphology. What is more, fibrinogen was specifically associated with a pro-inflammatory, pro-phagocytic microglial/macrophage phenotype, with a marked increase in CD68 expression relative to Iba-1. Indeed, a doubling of CD68 levels was seen in acute TBI despite little change in Iba-1, giving rise to an elevated CD68/Iba-1 ratio in the presence of fibrinogen. By contrast, there was no association between IgG deposition and microglial/macrophage activation in acute TBI.

The finding of increased CD68, but not Iba-1, in acute TBI is an interesting observation: as Iba-1 is an effective marker of overall microglial/macrophage number/density, our findings support the idea that a harmful inflammatory response in TBI does not arise through a simple expansion in cell number and a change in cell morphology. Instead, it is the specific activation of microglia/macrophages into cells with enhanced lysosomal and phagocytic capacity, that associates with tissue damage. This may explain the lack of benefit in TBI of non-specific immune suppression, suggesting that 
future translational studies focus on decreasing microglial/macrophage polarization to this specific phenotype. ${ }^{43}$ Indeed, this is one of the benefits of quantifying inflammatory proteins with known functions: it avoids having to make assumptions about microglial/macrophage activity based on morphology alone, and permits a more nuanced understanding of which of their roles are helpful, and which harmful, in TBI.

In long-term survival, a significant increase in CD68 and CD68/Iba-1 was seen in the corpus callosum and internal capsule. Significant numbers of amoeboid microglia/macrophages were also seen in the WM of the frontal lobe in a limited number of cases, and in the GM of the thalami. Such regional, chronic neuroinflammation has been commented on before in $\mathrm{TBI}$, but the reason for its restriction to specific brain regions is not yet known. ${ }^{27} 44$

Acute $\mathrm{TBI}$ was associated with a reduction in neuronal density, both at sites proximate to - and remote from - the injury nidus, confirming experimental findings. ${ }^{45,46}$ By contrast, no significant reduction in neuronal density was seen in long-term survival, where a reduction in cortical thickness was accompanied by a loss of the usually distinct pattern of cortical lamination. This finding is at odds with a prior report of neuronal loss in the hippocampal formation and thalamus in long-term survival. ${ }^{47}$ The discrepancy could relate to the small number of cases of long-term survival available to our study, but it is also feasible that long-term survivors are, by their nature, those with the least acute neuronal loss following injury. Indeed, evidence from animal models suggests that longterm survival from TBI is associated with a process of dendritic and synaptic degeneration, but not with overt neuronal loss. ${ }^{48,49}$ These features may be pertinent to the link between $\mathrm{TBI}$ and dementia, in which synaptic changes are also noted. Our findings require further work, including unbiased stereology on a larger cohort, to confirm the long-term fate of neurons following TBI. 
The reduction in neuronal density seen in acute TBI correlated significantly with fibrinogen deposition and increased CD68/Iba1, but not with parenchymal IgG. No relationship emerged between fibrinogen and neuronal density reduction in the brainstem, where fibrinogen deposition was minimal. Similarly, there was no relationship between brainstem neuronal density and CD68/Iba-1. A regression analysis indicated a strong, three-way interaction between increasing GM fibrinogen, increasing GM CD68/Iba1 , and decreasing neuronal density in acute TBI outside of the brainstem. An analysis of individual brain regions showed a weaker association in the frontal lobe compared with other areas, which may be a limitation of the small number of frontal lobe sections studied that had evidence of fibrinogen deposition. Alternatively, processes other than fibrinogen deposition (e.g. contusions and the presence of blood) may be important drivers of inflammation and reduced neuronal density in the frontal lobe. A study that uses sufficient contusion-containing material will help to shed light on this possibility.

Finally, we performed a trajectory analysis on fibrinogen gradients within individual cases of acute TBI, to assess for inflammation and neuronal density in regions of high and low fibrinogen. This strategy recapitulated the findings of the correlative and regression analyses, showing that, in an area of peak fibrinogen deposition, microglial activation is greatest, and neuronal density lowest. The trajectory analysis also helped mitigate against the possibility that the described associations were the result of tissue expansion alone. Where tissue is expanded, for example by microglial infiltration, neuronal density might be diminished without there being a true loss of neurons. However, if tissue expansion of the parenchyma is significant, it would be expected to reduce the density of fibrinogen staining as well: a feature not seen in the trajectory analysis. Furthermore, the deposition of IgG would also be expected to cause tissue expansion, and thus IgG deposition should also correlate with a reduction in neuronal density. This is contrary to our findings. Overall, whilst an important consideration, we believe that tissue expansion alone cannot explain the association between fibrinogen, inflammation and neuronal density. 
It is noteworthy that fibrinogen, but not IgG deposition, showed an association with CD68/Iba-1 and neuronal density. The putative interaction between fibrinogen, inflammation and neuronal density has been explained by the experimental binding of fibrinogen to the microglial/macrophage $\mathrm{CD} 11 \mathrm{~b} / \mathrm{CD} 18$ integrin receptor, with the subsequent upregulation of pro-inflammatory intracellular cascades, including the NF- $\kappa \mathrm{B}$ pathway. NF-KB generates tumour necrosis factor $\alpha$ and interleukin $1 \beta$, both of which promote glutamate-mediated neurotoxicity: a key pathological feature of acute TBI. ${ }^{11,50}$ That fibrinogen deposition in $A D$ and $M S$ is associated with inflammation and neuronal damage makes it feasible that fibrinogen plays a similar role in TBI. ${ }^{17}$

Our findings provide the first evidence of an association between fibrinogen, inflammation and reduced neuronal density in human TBI, and are rare insofar as they employ quantitative data to seek out potentially causal relationships in TBI in human postmortem tissue. However, one should not assume causality based on IHC findings alone, as these are only capable of providing a static snapshot of a dynamic process. Alternative explanations for the relationships that we report include fibrinogen being a surrogate marker of other, unmeasured features of acute TBI; the most obvious contender being vasogenic oedema. Whilst the areas of highest fibrinogen deposition were not those with greatest expansion in extracellular space, this does not rule out a role for oedema in the reduced neuronal density, as it can cause cell lysis. ${ }^{31}$ Alternatively, our findings could be interpreted as showing fibrinogen deposition as a result of BBB opening. This could occur in order in response to resident microglia signaling the presence of dead or dying neurons, which would be expected to increase the translocation of peripheral macrophages into the CNS. In this instance, the deposition of fibrinogen, and increase in microglia/macrophage activity, is the result of neuronal death prompting a clear up process. To provide a definitive answer about the provenance of parenchymal fibrinogen, and its temporal relationship to the activation of inflammatory cells and neuronal density, will require a means for assessing each factor longitudinally, be that experimental, or through combined imaging/post-mortem tissue analysis in humans. Should a role for fibrinogen in the activation of microglia/macrophages and the subsequent loss of neurons be confirmed, 
efforts could be taken to impair the fibrinogen/microglial interaction with the aim of preserving neuronal density.

Outside of the GM, our study identified significant axonal injury in acute TBI, especially in the large WM tracts of the corpus callosum, internal capsule and frontal lobe, as has previously been reported. ${ }^{51}$ Despite its abundance, axonal injury did not correlate with fibrinogen or IgG deposition, a finding upheld by trajectory analysis. Despite measures to exclude axonal profiles of $\mathrm{HII}$, it remains possible that axons accruing bAPP through processes other than trauma (e.g. Wallerian degeneration) were included in this count. However, our findings accord with previous studies of acute TBI, in which an increase is seen in tissue microglia/macrophage numbers, but in which there is no clear aggregation around, or interaction with, injured axons. ${ }^{52}$ Whilst reports exist of microglia interacting with damaged axons in acute TBI, they relate either to mild injury, which may differ pathologically, or the reports suggest that such interactions are few in number, with most microglia found next to WM capillaries and away from axons, much as we found. ${ }^{53} 54$ Experimentally reducing microglia does nothing to alter axonal survival in acute trauma, which supports the findings of others who show that neurons are the main target of acute microglial activation. ${ }^{55}$ Furthermore, axotomy could conceivably occur without an inflammatory response, as primary shear stresses and a host of secondary degenerative processes (e.g. axolemmal poration and filament damage, ionic disturbances, and intraaxonal transport failure) may be sufficient to explain $\beta$ APP accrual. ${ }^{57,58}$

No overall increase was seen in BAPP staining with long-term survival. However, one third of cases of long-term survival had evidence of scattered axonal spheroids, most notably in the pathologically thin sections of corpus callosum, and in the internal capsule, and these were co-located with perivascular fibrinogen. These areas also had an abundance of CD68-positive microglia/macrophages, a finding that has been reported 
elsewhere. ${ }^{60,61}$ The mechanism of sustained inflammation limited to white matter tracts, accompanied, as it is, by axonal transection, remains unclear. However, our observations suggest that it could be linked to chronic fibrinogen deposition. ${ }^{62}$ Of note, no microglial/macrophage clustering was seen around vessels lacking fibrinogen. Similarly, there was no spatial overlap between inflammation and axonal spheroids when perivascular IgG occurred without concomitant fibrinogen deposition. This mirrors findings made in other disease models, where fibrinogen-induced perivascular clustering of microglia/macrophages is a prerequisite of axonal transection. ${ }^{12}$ Whilst the contribution of fibrinogen-induced clustering to axonal injury is likely to be small in acute TBI, it may be a significant contributor to ongoing axonal loss in cases of long-term survival, and is worthy of a larger-scale study of fibrinogen deposition, microglial/macrophage activation, and axonal injury in long-term survival.

In summary, our study demonstrates:

- $\quad$ An increase in fibrinogen and IgG deposition in acute TBI, with important differences in location and abundance from those previously reported;

- A strong statistical and spatial relationship between GM fibrinogen deposition, GM microglial/macrophage activation, and reduced neuronal density in acute TBI. This requires additional work, ideally employing stereological neuronal counts, to further define the effect that fibrinogen and inflammation have on neuronal numbers;

- No relationship between IgG deposition, microglial/macrophage activation, and neuronal density;

- No overall relationship between WM fibrinogen/WM IgG and WM microglial/macrophage activation or $\beta$ APP staining;

- Rare foci of WM perivascular fibrinogen deposits, with attendant microglia/macrophage clustering, and $\beta$ APP-positive axonal profiles, in cases of acute and long-term survival. 
Our study suggests that fibrinogen may play a role in augmenting and sustaining an inflammatory state in TBI that impacts negatively on neuronal density and, potentially, axonal survival. This is in keeping with observations made in diseases such as AD and MS, where parenchymal fibrinogen is an important determinant of the inflammatory milieu, and where its removal decreases neuronal and axonal injury. ${ }^{17,12,63,64}$ This should prompt further study of the role played by fibrinogen in human TBI.

\section{AUTHOR CONTRIBUTIONS}

DRJ performed the experiments and data analysis, and wrote the manuscript. MJC and MME reviewed images and helped with the interpretation of results and reviewed the manuscript. GDL helped design the project, reviewed images, discussed the results, and helped in the writing of the manuscript.

\section{ACKNOWLEDGMENTS}

We wish to acknowledge the Oxford Brain Bank, (supported by the Medical Research Council (MRC), Brains for Dementia Research (BDR), and the NIHR Oxford Biomedical Research Centre) for the contribution of tissue. We are further indebted to Carolyn Sloan, Marie Hamard, and Connor Scott for their assistance in processing tissue.

\section{AUTHOR DISCLOSURE STATEMENT}

D.R. Jenkins and M.M. Esiri report no competing financial interests. G.C. DeLuca is supported by the NIHR Biomedical Research Centre (BRC), Oxford and has research 
Page 29 of 58

funding from the Oxford BRC, MRC(UK), and Merck-Serono. G.C. DeLuca has received travel expenses from Bay Schering, Biogen Idec, Genzyme, Merck Serono, and Novartis,

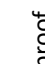
and honoraria as an invited speaker for Bayer Schering and Novartis. M.J. Craner has

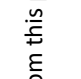
received travel expenses and honoraria from Biogen Idec, Genzyme, Novartis, Merck Serono, and Teva. 


\section{REFERENCES}

1. Saw, M.M., Chamberlain, J., Barr, M., Morgan, M.P.G., Burnett, J.R., and Ho, K.M. (2014). Differential Disruption of Blood-Brain Barrier in Severe Traumatic Brain Injury. Neurocrit Care 20, 209-216.

2. Hay, J.R., Johnson, V.E., Young, A.M.H., Smith, D.H., and Stewart, W. (2015). BloodBrain Barrier Disruption Is an Early Event That May Persist for Many Years After Traumatic Brain Injury in Humans. J Neuropathol Exp Neurol 74, 1147-1157.

3. Peeters, W., van den Brande, R., Polinder, S., Brazinova, A., Steyerberg, E.W., Lingsma, H.F., and Maas, A.I.R. (2015). Epidemiology of traumatic brain injury in Europe. Acta Neurochir (Wien) 157, 1683-1696.

4. Kinoshita, K. (2016). Traumatic brain injury: pathophysiology for neurocritical care. J Intensive Care 4, 1028-10.

5. Marmarou, A., Nichols, J., Burgess, J., Newell, D., Troha, J., Burnham, D., and Pitts, L. (1999). Effects of the bradykinin antagonist Bradycor (deltibant, CP-1027) in severe traumatic brain injury: results of a multi-center, randomized, placebo-controlled trial. American Brain Injury Consortium Study Group. J Neurotrauma 16, 431-444.

6. Morris, G.F., Bullock, R., Marshall, S.B., Marmarou, A., Maas, A., and Marshall, L.F. (1999). Failure of the competitive N-methyl-D-aspartate antagonist Selfotel (CGS 19755 ) in the treatment of severe head injury: results of two phase III clinical trials. The Selfotel Investigators. J Neurosurg 91, 737-743.

7. Edwards, P., Arango, M., Balica, L., Cottingham, R., El-Sayed, H., Farrell, B., Fernandes, J., Gogichaisvili, T., Golden, N., Hartzenberg, B., Husain, M., Ulloa, M.I., Jerbi, Z., Khamis, H., Komolafe, E., Laloë, V., Lomas, G., Ludwig, S., Mazairac, G., Muñoz Sanchéz, M. de L.A., Nasi, L., Olldashi, F., Plunkett, P., Roberts, I., Sandercock, P., Shakur, H., Soler, C., Stocker, R., Svoboda, P., Trenkler, S., Venkataramana, N.K., Wasserberg, J., Yates, D., Yutthakasemsunt, S., CRASH trial 
collaborators. (2005). Final results of MRC CRASH, a randomised placebo-controlled trial of intravenous corticosteroid in adults with head injury-outcomes at 6 months. Lancet 365, 1957-1959.

8. Shlosberg, D., Benifla, M., Kaufer, D., and Friedman, A. (2010). Blood-brain barrier breakdown as a therapeutic target in traumatic brain injury. Nat Rev Neurol 6, 393403.

9. Tomkins, O., Shelef, I., Kaizerman, I., Eliushin, A., Afawi, Z., Misk, A., Gidon, M., Cohen, A., Zumsteg, D., and Friedman, A. (2008). Blood-brain barrier disruption in post-traumatic epilepsy. J Neurol Neurosurg Psychiatry 79, 774-777.

10. Chodobski, A., Zink, B.J., and Szmydynger-Chodobska, J. (2011). Blood-Brain Barrier Pathophysiology in Traumatic Brain Injury. Transl Stroke Res 2, 492-516.

11. Flick, M.J., Du, X., Witte, D.P., Jiroušková, M., Soloviev, D.A., Busuttil, S.J., Plow, E.F., and Degen, J.L. (2004). Leukocyte engagement of fibrin(ogen) via the integrin receptor alphaMbeta2/Mac-1 is critical for host inflammatory response in vivo. J Clin Invest 113, 1596-1606.

12. Davalos, D., Ryu, J.K., Merlini, M., Baeten, K.M., Le Moan, N., Petersen, M.A., Deerinck, T.J., Smirnoff, D.S., Bedard, C., Hakozaki, H., Gonias Murray, S., Ling, J.B., Lassmann, H., Degen, J.L., Ellisman, M.H., and Akassoglou, K. (2012). Fibrinogeninduced perivascular microglial clustering is required for the development of axonal damage in neuroinflammation. Nat Commun 3, 1227.

13. Viggars, A.P., Wharton, S.B., Simpson, J.E., Matthews, F.E., Brayne, C., Savva, G.M., Garwood, C., Drew, D., Shaw, P.J., and Ince, P.G. (2011). Alterations in the blood brain barrier in ageing cerebral cortex in relationship to Alzheimer-type pathology: A study in the MRC-CFAS population neuropathology cohort. Neurosci Lett 505, 2530.

14. Cortes-Canteli, M., Paul, J., Norris, E.H., Bronstein, R., Ahn, H.J., Zamolodchikov, D., Bhuvanendran, S., Fenz, K.M., and Strickland, S. (2010). Fibrinogen and beta-amyloid 
association alters thrombosis and fibrinolysis: a possible contributing factor to Alzheimer's disease. Neuron 66, 695-709.

15. Liu, J.Y.W., Thom, M., Catarino, C.B., Martinian, L., Figarella-Branger, D., Bartolomei, F., Koepp, M., and Sisodiya, S.M. (2012). Neuropathology of the blood-brain barrier and pharmaco-resistance in human epilepsy. Brain 135, 3115-3133.

16. Akassoglou, K., Yu, W.M., Akpinar, P., and Strickland, S. (2002). Fibrin inhibits peripheral nerve remyelination by regulating Schwann cell differentiation. Neuron $33,861-875$.

17. Ryu, J.K., and McLarnon, J.G. (2009). A leaky blood-brain barrier, fibrinogen infiltration and microglial reactivity in inflamed Alzheimer's disease brain. J Cell Mol Med 13, 2911-2925.

18. Akassoglou, K., Adams, R.A., Bauer, J., Mercado, P., Tseveleki, V., Lassmann, H., Probert, L., and Strickland, S. (2004). Fibrin depletion decreases inflammation and delays the onset of demyelination in a tumor necrosis factor transgenic mouse model for multiple sclerosis. Proc Natl Acad Sci USA 101, 6698-6703.

19. Patibandla, P.K., Tyagi, N., Dean, W.L., Tyagi, S.C., Roberts, A.M., and Lominadze, D. (2009). Fibrinogen induces alterations of endothelial cell tight junction proteins. J Cell Physiol 221, 195-203.

20. Tyagi, N., Roberts, A.M., Dean, W.L., Tyagi, S.C., and Lominadze, D. (2007). Fibrinogen induces endothelial cell permeability. Mol Cell Biochem 307, 13-22.

21. Webster, K.M., Sun, M., Crack, P., O’Brien, T.J., Shultz, S.R., and Semple, B.D. (2017). Inflammation in epileptogenesis after traumatic brain injury. J Neuroinflammation $14,1-17$.

22. Malec, J.F., Brown, A.W., Leibson, C.L., Flaada, J.T., Mandrekar, J.N., Diehl, N.N., and Perkins, P.K. (2007). The Mayo Classification System for Traumatic Brain Injury Severity. J Neurotrauma 24, 1417-1424. 
23. Teasdale, G., and Jennett, B. (1974). Assessment of coma and impaired consciousness. A practical scale. Lancet 2, 81-84.

24. Gill, S.K., Ishak, M., and Rylett, R.J. (2005). Exposure of nuclear antigens in formalinfixed, paraffin-embedded necropsy human spinal cord tissue: Detection of NeuN. J Neurosci Methods 148, 26-35.

25. McAllister, T.W. (2011). Neurobiological consequences of traumatic brain injury. Dialogues Clin Neurosci 13, 287-300.

26. Yates, R.L., Esiri, M.M., Palace, J., Jacobs, B., Perera, R., and DeLuca, G.C. (2017). Fibrin(ogen) and neurodegeneration in the progressive multiple sclerosis cortex. Ann Neurol 82, 259-270.

27. Johnson, V.E., Stewart, J.E., Begbie, F.D., Trojanowski, J.Q., Smith, D.H., and Stewart, W. (2013). Inflammation and white matter degeneration persist for years after a single traumatic brain injury. Brain 136, 28-42.

28. Geddes, J.F., Vowles, G.H., Beer, T.W., and Ellison, D.W. (1997). The diagnosis of diffuse axonal injury: implications for forensic practice. Neuropathol Appl Neurobiol $23,339-347$.

29. Hayashi, T., Ago, K., Nakamae, T., Higo, E., and Ogata, M. (2015). Two different immunostaining patterns of beta-amyloid precursor protein (APP) may distinguish traumatic from nontraumatic axonal injury. Int J Legal Med 129, 1085-1090.

30. Korzhevskii, D.E., and Kirik, O.V. (2016). Brain Microglia and Microglial Markers. Neurosci Behav Physi 46, 284-290.

31. Klatzo, I. (1967). Presidental address. Neuropathological aspects of brain edema. J Neuropathol Exp Neurol 26, 1-14.

32. Ünal-Çevik, I., Kılınç, M., Gursoy-Ozdemir, Y., Gurer, G., and Dalkara, T. (2004). Loss of NeuN immunoreactivity after cerebral ischemia does not indicate neuronal cell loss: a cautionary note. Brain Res 1015, 169-174. 
33. Lavezzi, A.M., Corna, M.F., and Matturri, L. (2013). Neuronal nuclear antigen (NeuN): A useful marker of neuronal immaturity in sudden unexplained perinatal death. J Neurol Sci 329, 45-50.

34. Chodobski, A., Zink, B.J., and Szmydynger-Chodobska, J. (2011). Blood-Brain Barrier Pathophysiology in Traumatic Brain Injury. Transl Stroke Res 2, 492-516.

35. Habgood, M.D., Bye, N., Dziegielewska, K.M., Ek, C.J., Lane, M.A., Potter, A., Morganti-Kossmann, C., and Saunders, N.R. (2007). Changes in blood-brain barrier permeability to large and small molecules following traumatic brain injury in mice. Eur J Neurosci 25, 231-238.

36. Zobel, K., Hansen, U., and Galla, H.-J. (2016). Blood-brain barrier properties in vitro depend on composition and assembly of endogenous extracellular matrices. Cell Tissue Res. 365, 1-13.

37. Gentry, L.R., Thompson, B., and Godersky, J.C. (1988). Trauma to the corpus callosum: MR features. AJNR Am J Neuroradiol 9, 1129-1138.

38. Abdel-Dayem, H.M., Abu-Judeh, H., Kumar, M., Atay, S., Naddaf, S., El-Zeftawy, H., and Luo, J.Q. (1998). SPECT brain perfusion abnormalities in mild or moderate traumatic brain injury. Clin Nucl Med 23, 309-317.

39. Maxwell, W.L. (2004). Differential responses in three thalamic nuclei in moderately disabled, severely disabled and vegetative patients after blunt head injury. Brain $127,2470-2478$.

40. Ross, D.T., Graham, D.I., and Adams, J.H. (1993). Selective loss of neurons from the thalamic reticular nucleus following severe human head injury. J Neurotrauma 10, 151-165.

41. Little, D.M., Kraus, M.F., Joseph, J., Geary, E.K., Susmaras, T., Zhou, X.J., Pliskin, N., and Gorelick, P.B. (2010). Thalamic integrity underlies executive dysfunction in traumatic brain injury. Neurology 74, 558-564. 
42. Klatzo, I., Wiśniewski, H., and Smith, D.E. (1965). Observations on penetration of serum proteins into the central nervous system. Prog Brain Res 15, 73-88.

43. Maas, A.I.R., Stocchetti, N., and Bullock, R. (2008). Moderate and severe traumatic brain injury in adults. Lancet neurol 7, 728-741.

44. Ramlackhansingh, A.F., Brooks, D.J., Greenwood, R.J., Bose, S.K., Turkheimer, F.E., Kinnunen, K.M., Gentleman, S., Heckemann, R.A., Gunanayagam, K., Gelosa, G., and Sharp, D.J. (2011). Inflammation after trauma: Microglial activation and traumatic brain injury. Ann Neurol 70, 374-383.

45. Hicks, R., Soares, H., Smith, D., and Mclntosh, T. (1996). Temporal and spatial characterization of neuronal injury following lateral fluid-percussion brain injury in the rat. Acta Neuropathol 91, 236-246.

46. Balança, B., Bapteste, L., Lieutaud, T., Ressnikoff, D., Guy, R., Bezin, L., and Marinesco, S. (2016). Neuronal loss as evidenced by automated quantification of neuronal density following moderate and severe traumatic brain injury in rats. J Neurosci Res 94, 39-49.

47. Smith, D.H., Johnson, V.E., and Stewart, W. (2013). Chronic neuropathologies of single and repetitive TBI: substrates of dementia? Nat Rev Neurol 9, nrneurol.2013.29-221.

48. Gao, X., and Chen, J. (2011). Mild Traumatic Brain Injury Results in Extensive Neuronal Degeneration in the Cerebral Cortex. J Neuropathol Exp Neurol 70, 183191.

49. Johnson, V.E., and Stewart, W. (2015). Traumatic brain injury: Age at injury influences dementia risk after TBI. Nat Rev Neurol 11, 128-130.

50. Ye, L., Huang, Y., Zhao, L., Li, Y., Sun, L., Zhou, Y., Qian, G., and Zheng, J.C. (2013). IL$1 \beta$ and TNF- $\alpha$ induced neurotoxicity through glutamate production: a potential role for neuronal glutaminase. J. Neurochem. 125, 897-908. 
51. Adams, H., Mitchell, D.E., Graham, D.I., and Doyle, D. (1977). Diffuse brain damage of immediate impact type. Its relationship to"primary brain-stem damage" in head injury. Brain 100, 489-502.

52. Kelley, B.J., Lifshitz, J., and Povlishock, J.T. (2007). Neuroinflammatory responses after experimental diffuse traumatic brain injury. J Neuropathol Exp Neurol 66, 9891001.

53. Lafrenaye, A.D., Todani, M., Walker, S.A., and Povlishock, J.T. (2015). Microglia processes associate with diffusely injured axons following mild traumatic brain injury in the micro pig. J Neuroinflammation, 1-15.

54. Shitaka, Y., Tran, H.T., Bennett, R.E., Sanchez, L., Levy, M.A., Dikranian, K., and Brody, D.L. (2011). Repetitive closed-skull traumatic brain injury in mice causes persistent multifocal axonal injury and microglial reactivity. J Neuropathol Exp Neurol 70, 551-567.

55. Wofford, K.L., Harris, J.P., Browne, K.D., Brown, D.P., Grovola, M.R., Mietus, C.J., Wolf, J.A., Duda, J.E., Putt, M.E., Spiller, K.L., and Cullen, D.K. (2017). Rapid Neuroinflammatory Response Localized to Injured Neurons After Diffuse Traumatic Brain Injury in Swine. Exp Neurol, 1-34.

56. Bennett, R.E., and Brody, D.L. (2014). Acute Reduction of Microglia Does Not Alter Axonal Injury in a Mouse Model of Repetitive Concussive Traumatic Brain Injury. J Neurotrauma 31, 1647-1663.

57. Povlishock, J.T., and W, J.L. (1995). Are the pathobiological changes evoked by traumatic brain injury immediate and irreversible? Brain Pathol. 5, 415-426.

58. Hånell, A., Greer, J.E., McGinn, M.J., and Povlishock, J.T. (2015). Traumatic brain injury-induced axonal phenotypes react differently to treatment. Acta Neuropathol $129,317-332$. 
59. Hill, C.S., Coleman, M.P., and Menon, D.K. (2016). Traumatic Axonal Injury: Mechanisms and Translational Opportunities. Trends in Neurosciences, 1-14.

60. Chen, X.-H., Johnson, V.E., Uryu, K., Trojanowski, J.Q., and Smith, D.H. (2009). A Lack of Amyloid $\beta$ Plaques Despite Persistent Accumulation of Amyloid $\beta$ in Axons of Long-Term Survivors of Traumatic Brain Injury. Brain Pathol 19, 214-223.

61. Smith, D.H., Johnson, V.E., and Stewart, W. (2013). Chronic neuropathologies of single and repetitive TBI: substrates of dementia? Nature Publishing Group 9, 211221.

62. Johnson, V.E., Stewart, W., and Smith, D.H. (2013). Axonal pathology in traumatic brain injury. Exp Neurol 246, 35-43.

63. Adams, R.A., Bauer, J., Flick, M.J., Sikorski, S.L., Nuriel, T., Lassmann, H., Degen, J.L., and Akassoglou, K. (2007). The fibrin-derived $y$ 377-395peptide inhibits microglia activation and suppresses relapsing paralysis in central nervous system autoimmune disease. J Exp Med 204, 571-582.

64. Paul, J., Strickland, S., and Melchor, J.P. (2007). Fibrin deposition accelerates neurovascular damage and neuroinflammation in mouse models of Alzheimer's disease. J Exp Med 204, 1999-2008. 
Page 38 of 58

Table 1. Clinical and demographic information.

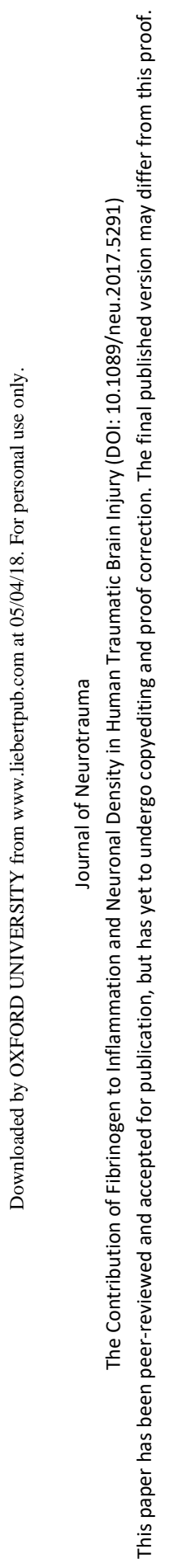


Page 39 of 58

Figure $1 \quad$ IgG and Fibrinogen Staining in Acute TBI

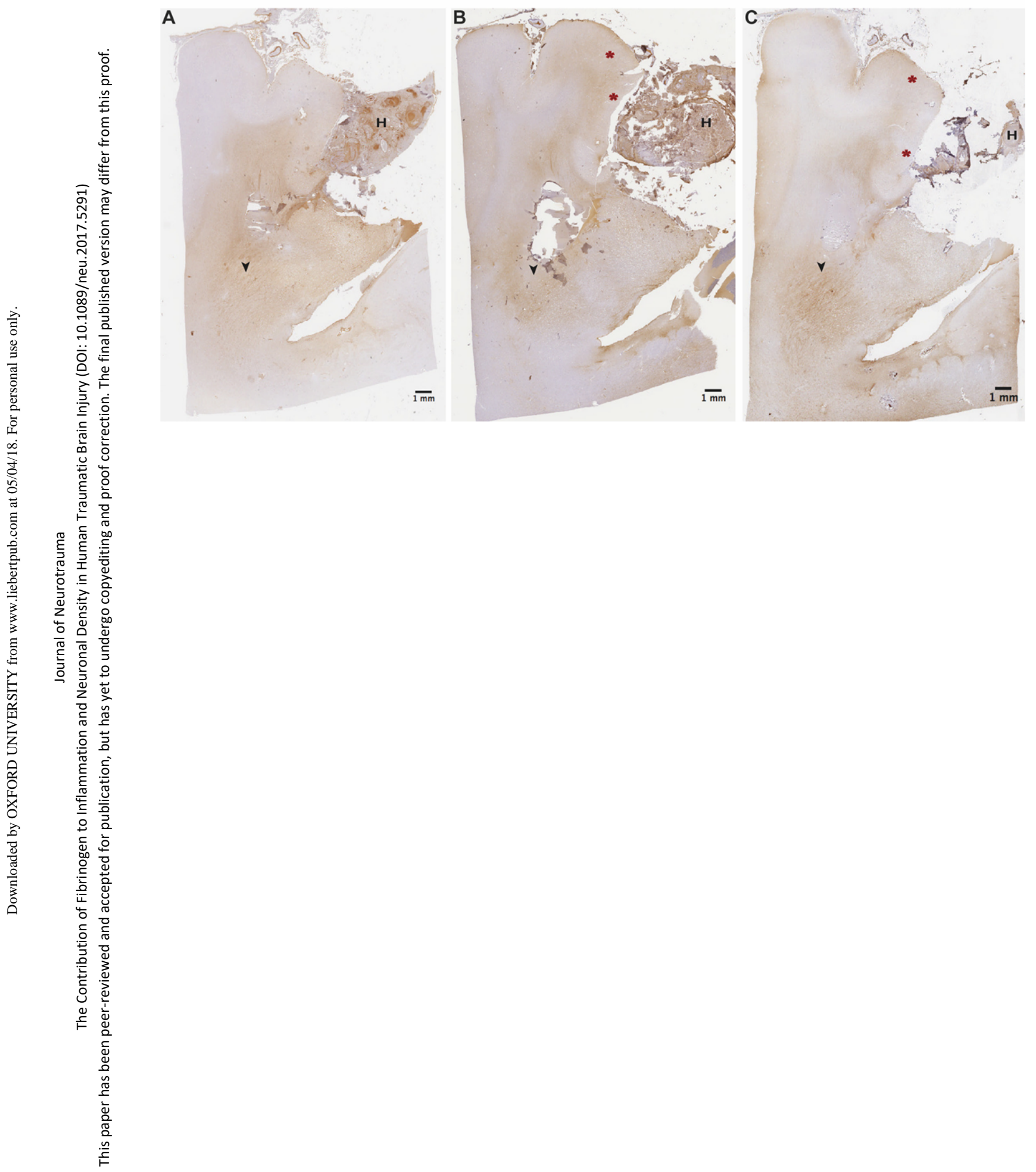


Figure $1 \quad$ IgG and Fibrinogen Staining in Acute TBI

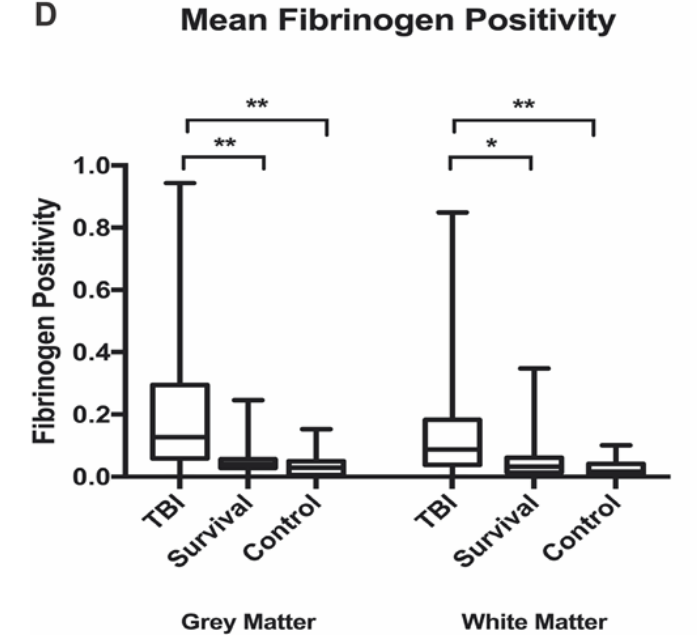

E Mean IgG Positivity

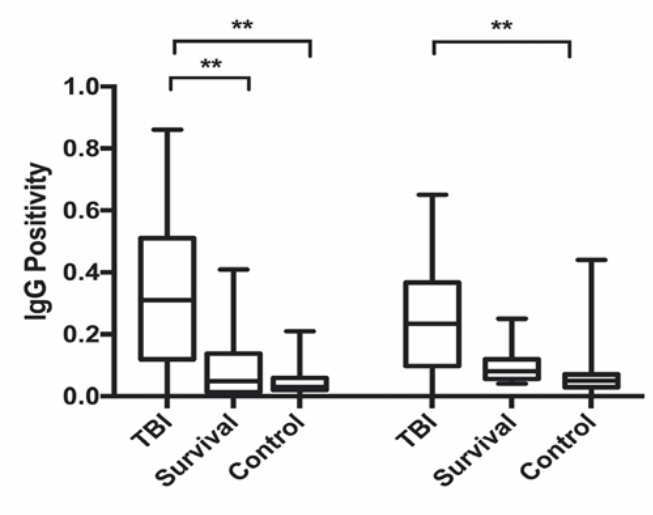

Grey Matter White Matter
40

Cohorts

$\mathrm{TBI}=$ Acute TBI

Survival $=$ Long-term survival

Control

Figure 1. In acute TBI, both IgG and fibrinogen are deposited in the brain parenchyma.

Within individual cases, fibrinogen and IgG deposition can overlap, or can be seen staining different compartments, e.g. IgG in the WM (A, arrow head) and fibrinogen in both the GM ( $B-C$, asterisk) and WM (B-C, arrowhead). This may represent differences in the rate at which each protein is cleared from the parenchyma. Similar staining patterns are produced by monoclonal (B) and polyclonal (C) antibodies against fibrinogen. In focal injury, IgG and fibrinogen overlap, as seen in an organising haematoma $(\mathrm{H})$ and next to a contusion (arrow heads). Fibrinogen deposition is significantly greater in acute TBI than in long-term survival or control. Graphs show GM fibrinogen (D) positivity (all brain regions combined) [mean (95\% confidence intervals) $]=0.20(0.15-0.21)$ vs $0.05(0.03-0.07)$ vs $0.04(0.03-0.05), P$ $<0.0001$; and WM positivity $=0.16(0.12-0.21)$ vs $0.05(0.02-0.08)$ vs $0.03(0.02-0.03), P$ $<0.0001$. IgG deposition is also significantly greater in acute TBI than in long-term survival or control (E): GM positivity $0.32(0.26-0.39)$ vs 0.09 (0.03-0.16) vs 0.05 (0.04-0.07), P $<0.0001$; WM positivity 0.24 (0.18-0.29) vs 0.07 (0.07-0.13) vs 0.07 (0.05-0.09), $\mathrm{P}<0.0001$. [Significance values: * $\mathrm{P}<0.05 ; * * \mathrm{P}<0.01$.] 
Page 41 of 58

Figure 2 Comparison of Fibrinogen Deposition in Acute TBI, Long-term Survival from TBI, and Control

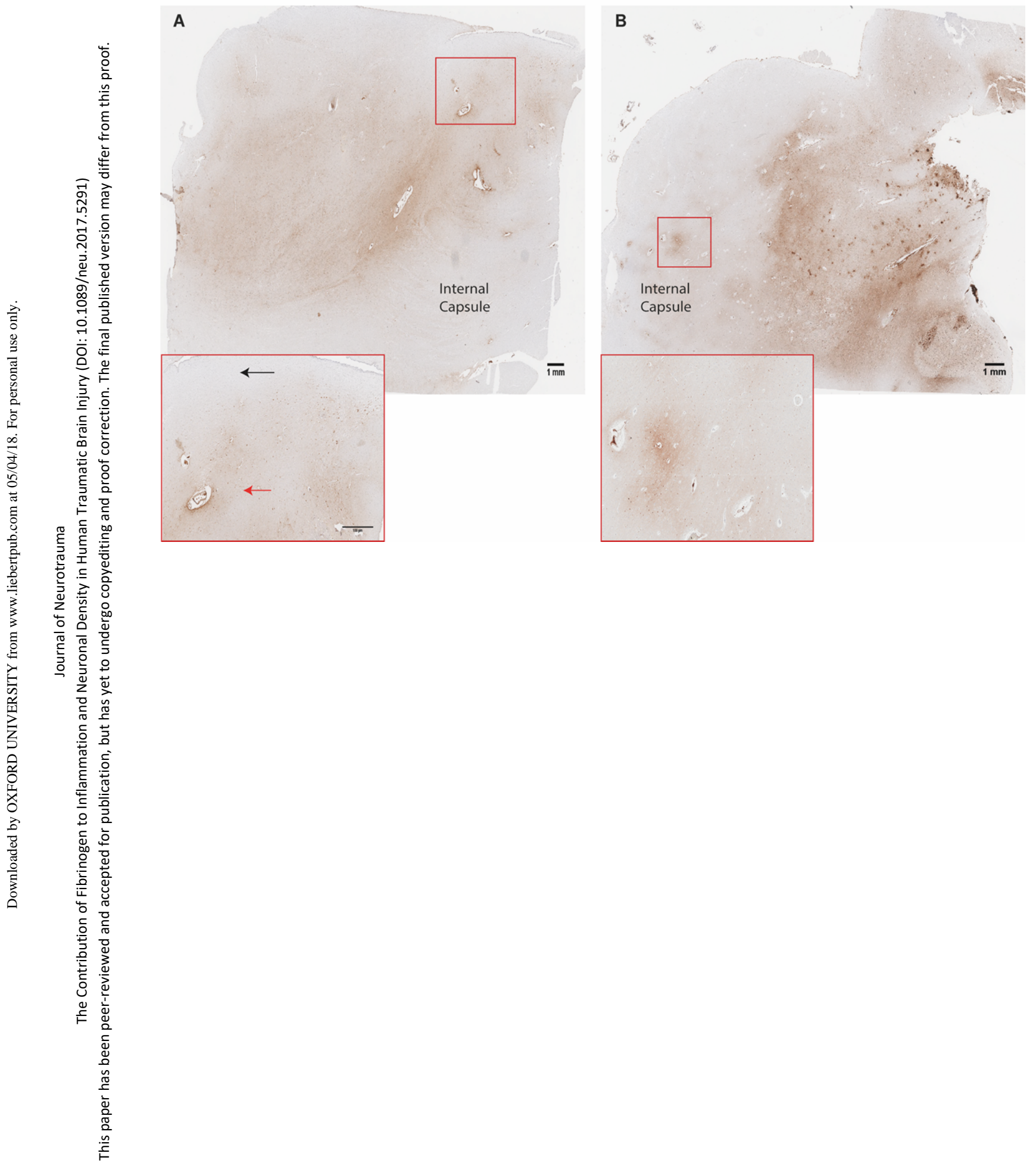


Page 42 of 58

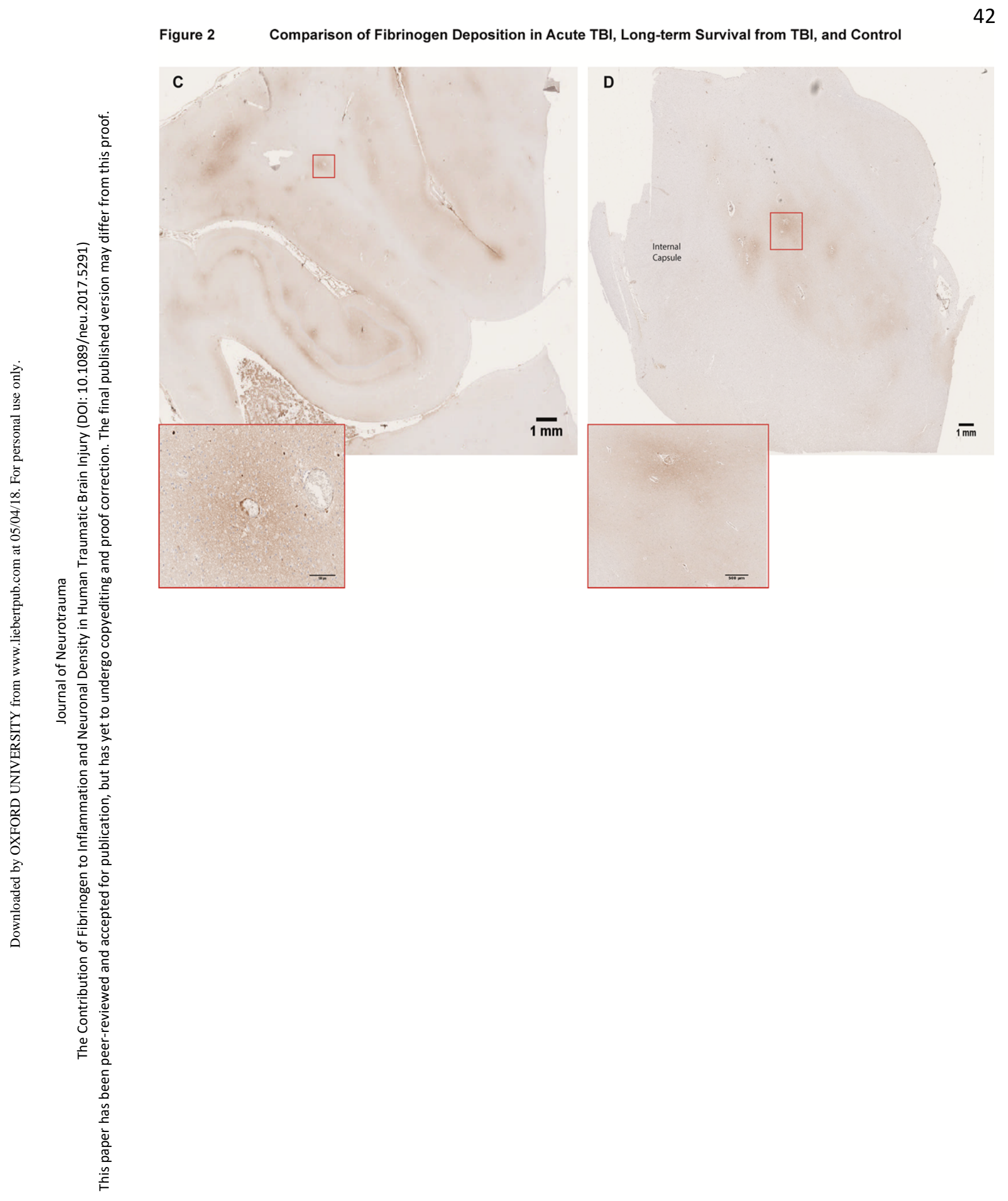


Figure 2

Comparison of Fibrinogen Deposition in Acute TBI, Long-term Survival from TBI, and Control

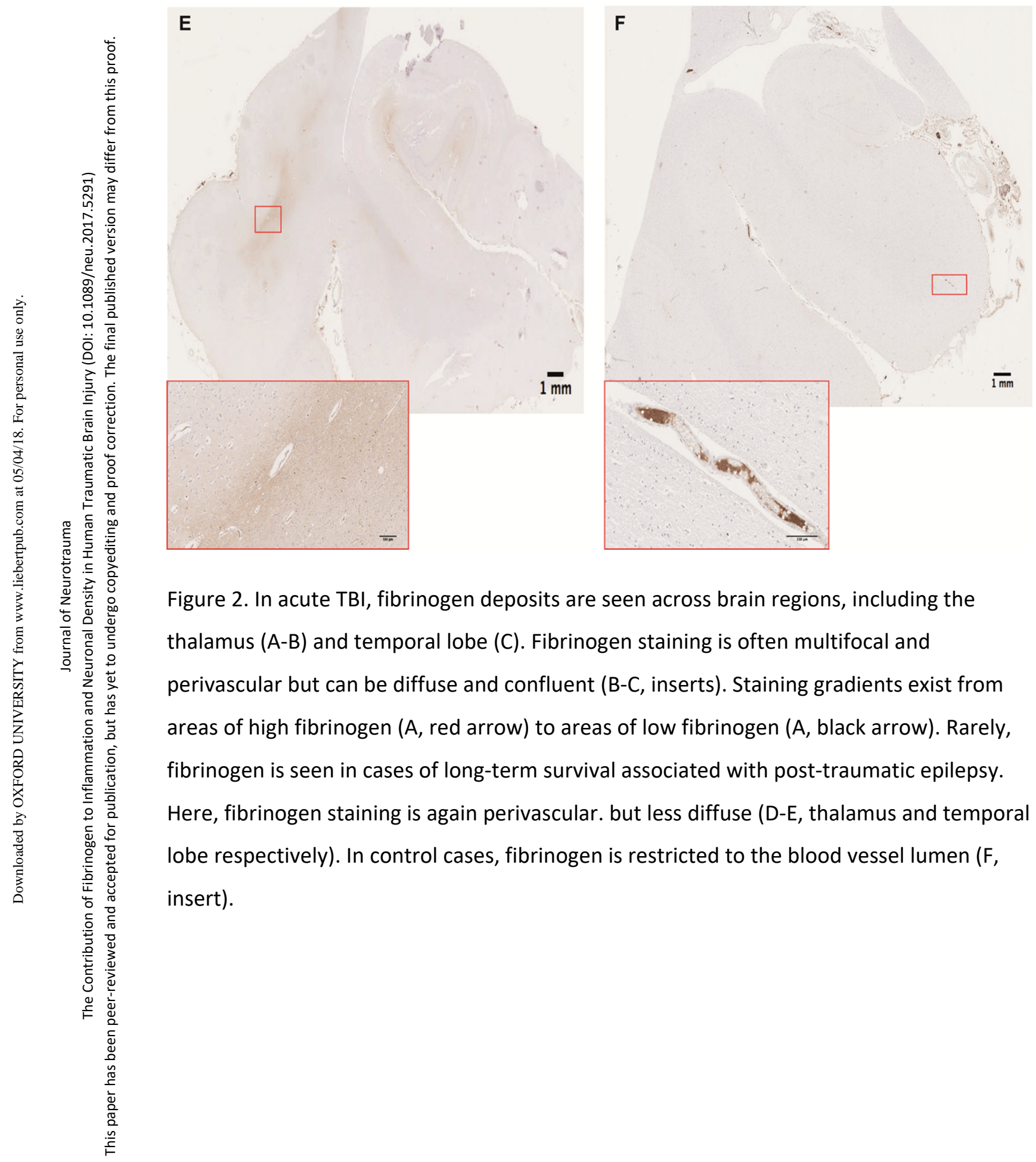


Page 44 of 58

Figure 3 Correlations in Acute TBI: Grey Matter Fibrinogen, CD68/lba-1, and Neurons/mm ${ }^{2}$

\section{(Brain areas combined)}

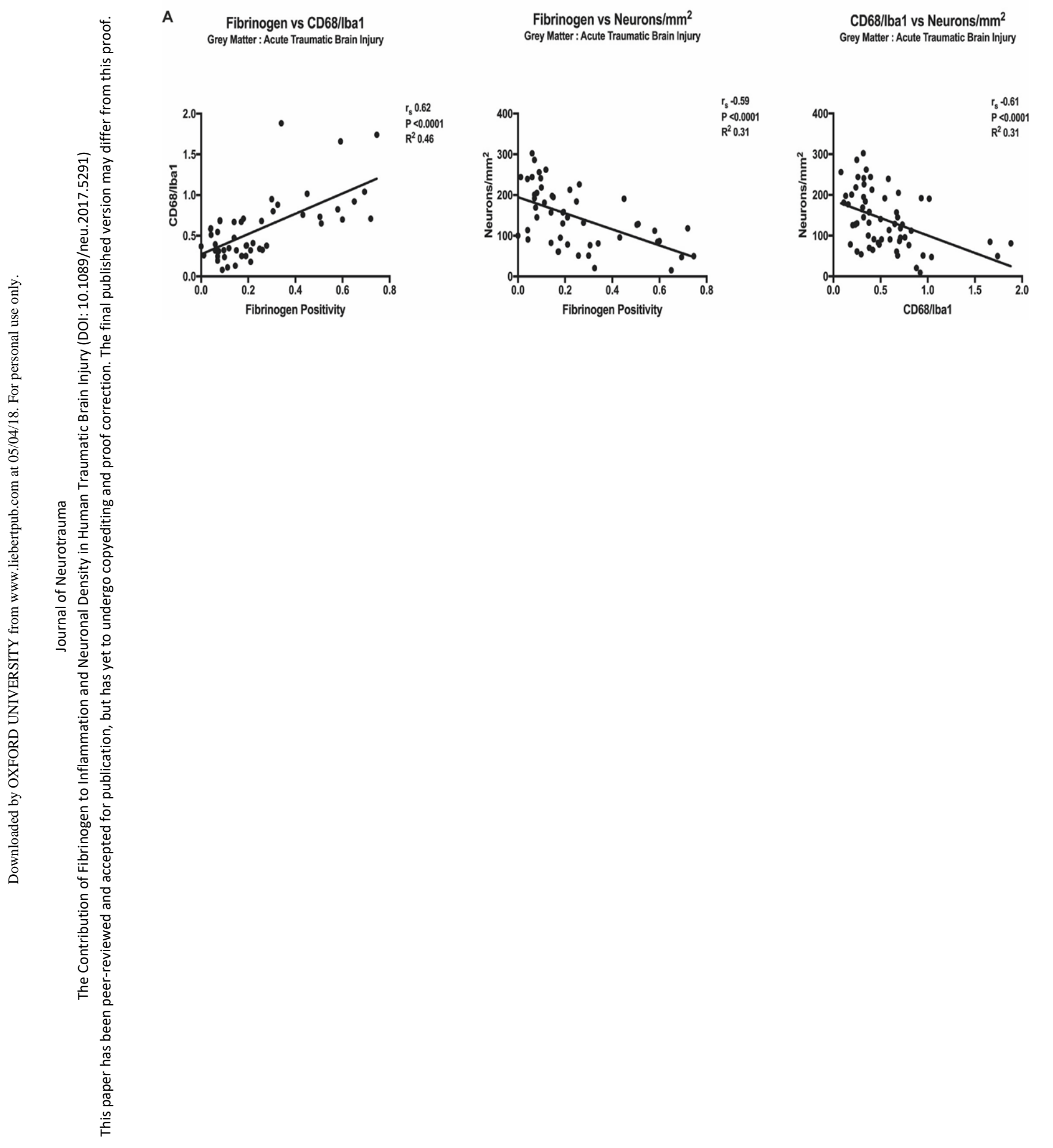


Figure 3 Correlations in Acute TBI: Grey Matter Fibrinogen, CD68/lba-1, and Neurons/mm ${ }^{2}$ (Brain areas combined)

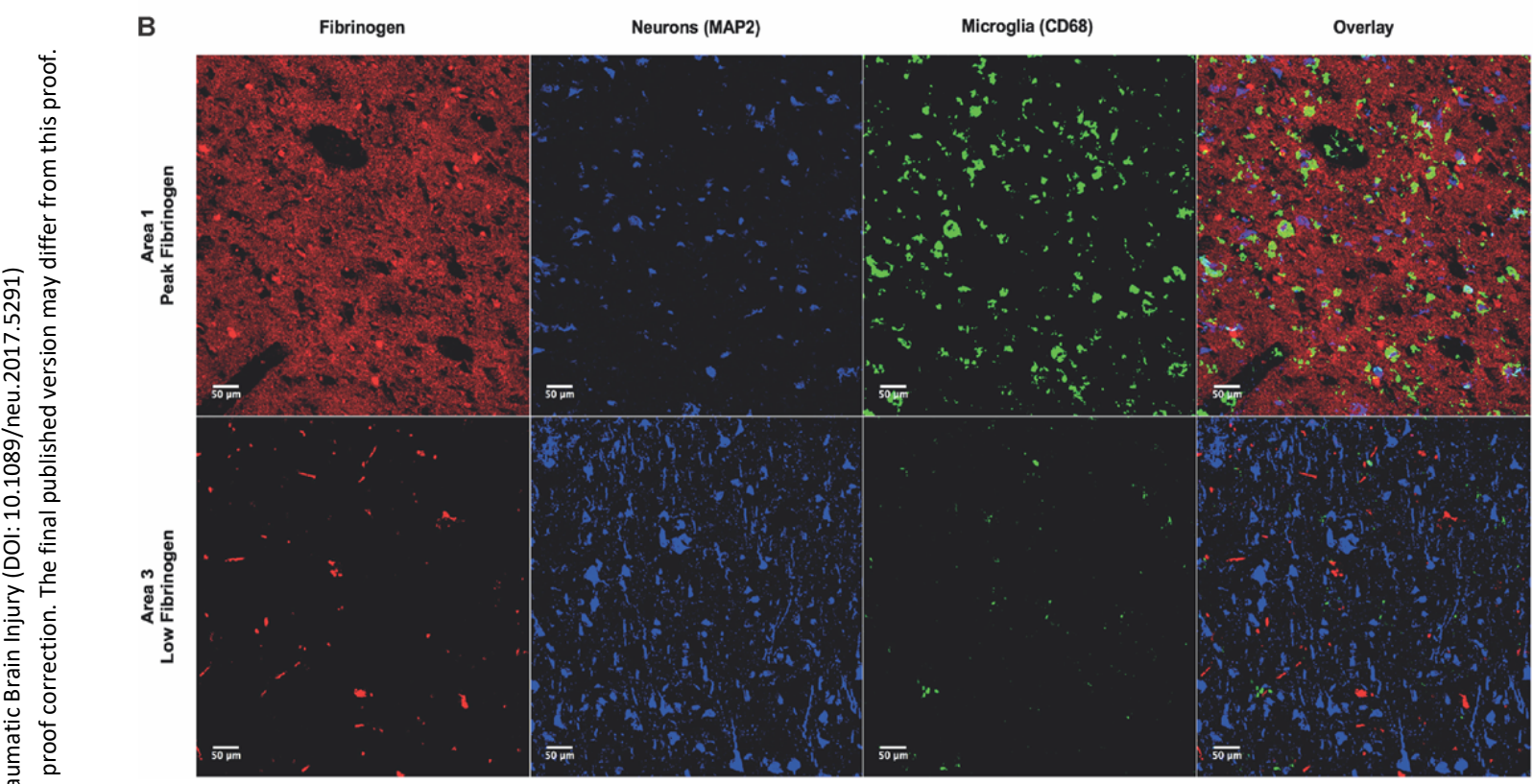

Figure 3. (A) Correlations between GM fibrinogen, CD68/lba-1 and neurons $/ \mathrm{mm}^{2}$ in acute TBI. (B) Immunofluorescence was used to examine the association between fibrinogen gradients, inflammation (shown here as CD68) and neuronal density (B). In areas of peak fibrinogen deposition (Area 1), microglia/macrophages are activated, and neurons are reduced in number. In the same tissue section, but in an area of low fibrinogen (Area 3), neuron density is greater, and fewer activated microglia/macrophages are seen. 


\begin{tabular}{|c|c|c|c|c|c|c|}
\hline Target & $\begin{array}{l}\text { Primary } \\
\text { Antibody }\end{array}$ & $\begin{array}{c}\text { Antibody } \\
\text { Concentration }\end{array}$ & $\begin{array}{l}\text { Antigen } \\
\text { Retrieval }\end{array}$ & $\begin{array}{c}\text { Blocking } \\
\text { Step }\end{array}$ & $\begin{array}{c}\text { Incubation } \\
\text { Settings }\end{array}$ & Positive Controls \\
\hline Fibrinogen (monoclonal) & $\begin{array}{l}\text { Abcam } \\
\text { ab58207 }\end{array}$ & $1 / 5000$ & AC Citrate & Nil & $4^{\circ} \mathrm{CON}$ & $\begin{array}{l}\text { Multiple sclerosis } \\
\text { tissue with known } \\
\text { staining. Also } \\
\text { compared with } \\
\text { polyclonal fibrinogen } \\
\text { antibody staining }\end{array}$ \\
\hline Fibrinogen (polyclonal) & $\begin{array}{l}\text { Dako } \\
\text { A0080 }\end{array}$ & $1 / 50,000$ & $\begin{array}{l}\text { AC Tris } \\
\text { EDTA }\end{array}$ & Nil & $4^{\circ} \mathrm{CON}$ & $\begin{array}{l}\text { Multiple sclerosis } \\
\text { tissue with known } \\
\text { staining. Also } \\
\text { compared with } \\
\text { monoclonal } \\
\text { fibrinogen antibody } \\
\text { staining }\end{array}$ \\
\hline Immunoglobulin G & $\begin{array}{l}\text { Dako } \\
\text { A0423 }\end{array}$ & $1 / 5000$ & Nil & Nil & RT 15 mins & Human tonsil \\
\hline Microglia/macrophages & Dako & $1 / 50$ & AC Citrate & Nil & RT 60 mins & Human tonsil \\
\hline
\end{tabular}




\begin{tabular}{|l|l|l|l|l|l|l|}
\hline (CD68) & M0876 & & & & \\
\hline $\begin{array}{l}\text { Microglia/macrophages } \\
\text { (Iba-1) }\end{array}$ & $\begin{array}{l}\text { Wako } \\
019-19741\end{array}$ & $1 / 1000$ & $\begin{array}{l}\text { AC Tris } \\
\text { EDTA }\end{array}$ & Nil & $4^{\circ} \mathrm{C}$ ON & Human tonsil \\
\hline Neurons (NeuN) & $\begin{array}{l}\text { Millipore } \\
\text { MAB377 }\end{array}$ & $1 / 100$ & $\begin{array}{l}\text { AC Tris } \\
\text { EDTA }\end{array}$ & $\begin{array}{l}10 \% \mathrm{FCS} \\
1 \text { hour }\end{array}$ & $4^{\circ} \mathrm{C}$ ON & Human brain \\
\hline Injured axons ( $\beta$ APP) & $\begin{array}{l}\text { Life } \\
\text { Technologies } \\
\text { LN27 }\end{array}$ & $1 / 10,000$ & AC Citrate & Nil & $4^{\circ} \mathrm{C}$ ON & $\begin{array}{l}\text { Injured human brain } \\
\text { identified by }\end{array}$ \\
\hline
\end{tabular}

$A C=$ autoclave $; \beta A P P=$ beta Amyloid Precursor Protein; FA = formic acid; FCS = fetal calf serum; lba- 1 = ionised calcium binding adaptor molecule-1; $\mathrm{MW}=$ microwave; $\mathrm{ON}=$ overnight; $\mathrm{RT}=$ room temperature. 
Page 48 of 58

48

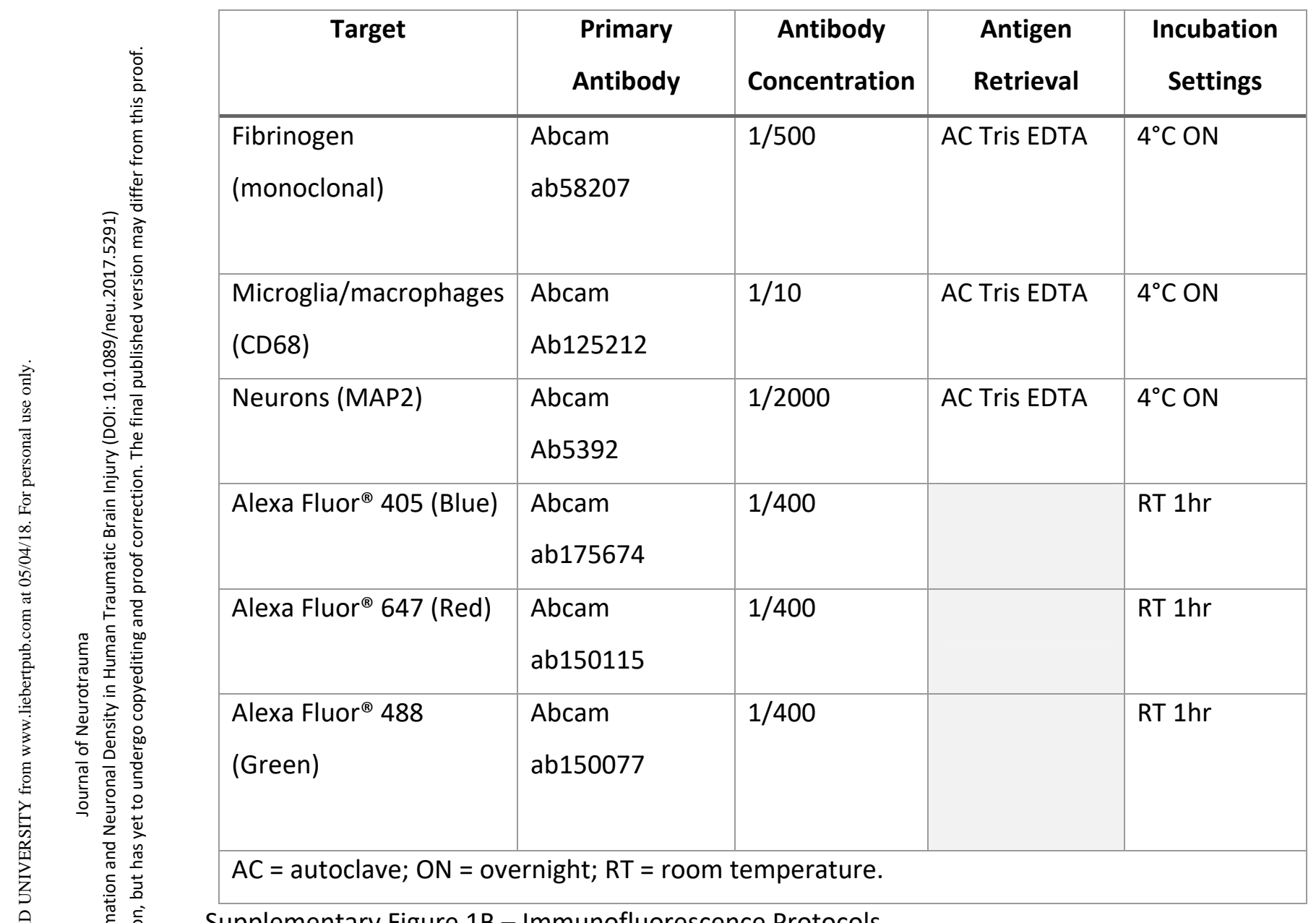

Supplementary Figure 1B - Immunofluorescence Protocols 
49

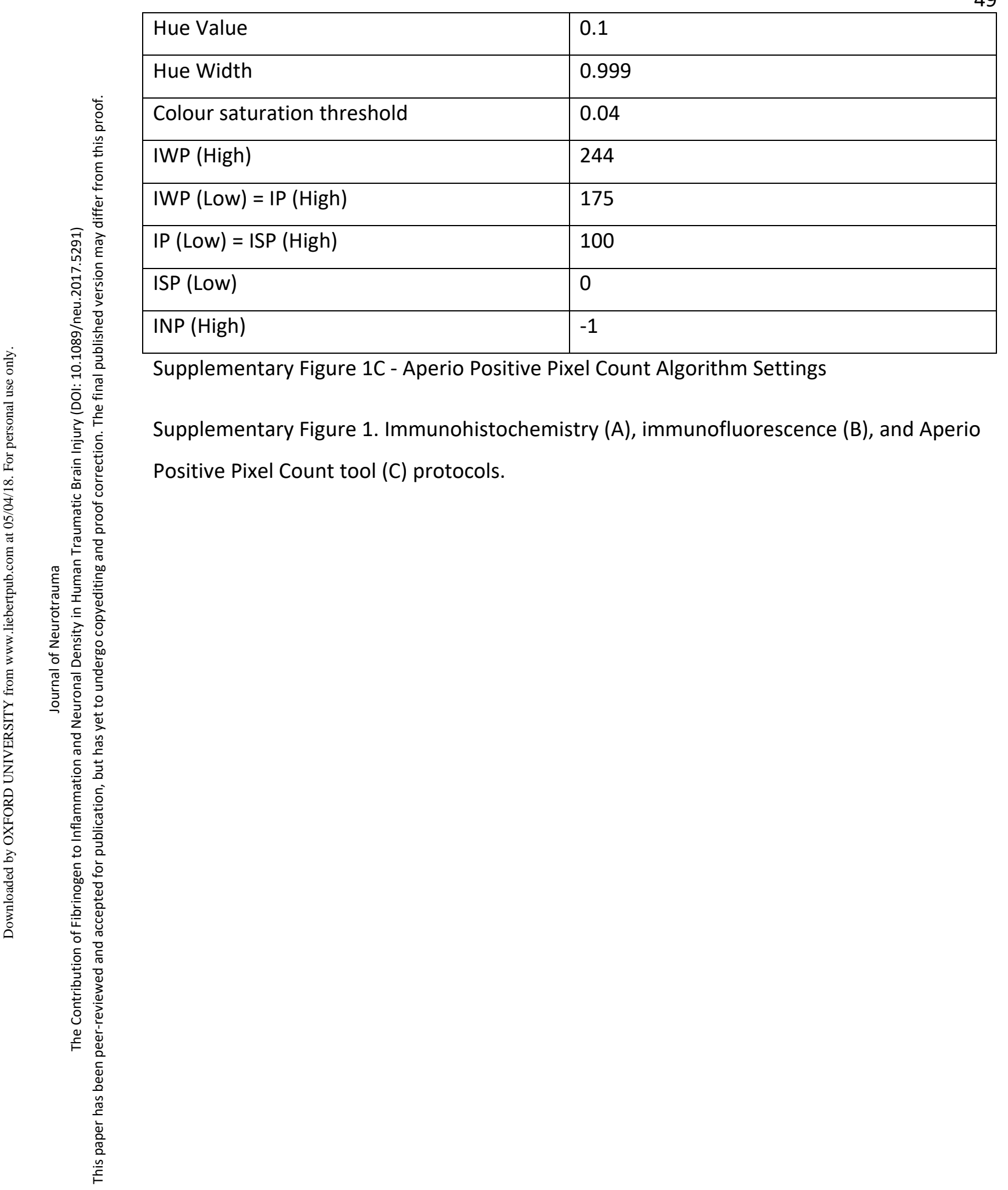




\begin{tabular}{|c|c|c|c|c|c|c|c|c|c|c|c|c|c|c|c|c|c|c|c|c|}
\hline \multirow{4}{*}{$\begin{array}{l}\text { TBI } \\
\text { Cas } \\
\text { e }\end{array}$} & \multirow{4}{*}{$\begin{array}{l}\text { Time } \\
\text { from } \\
\text { Injury to } \\
\text { Death } \\
\text { (days) }\end{array}$} & \multirow{4}{*}{$\begin{array}{l}\text { Post- } \\
\text { Mortem } \\
\text { Delay } \\
\text { (days) }\end{array}$} & \multirow{4}{*}{$\begin{array}{c}\text { Injury } \\
\text { Severity } \\
\text { (GCS/lesion) }\end{array}$} & \\
\hline & & & & \multicolumn{17}{|c|}{$\begin{array}{l}\text { 1. Grey box = protein present in tissue section } \\
\text { 2. Chequered box = no tissue section } \\
\text { 3. C = contusion in tissue section }\end{array}$} \\
\hline & & & & \multicolumn{2}{|c|}{$\begin{array}{l}\text { Frontal } \\
\text { Lobe }\end{array}$} & \multicolumn{2}{|c|}{$\begin{array}{c}\text { Tempora } \\
\text { I Lobe }\end{array}$} & \multicolumn{2}{|c|}{$\begin{array}{l}\text { Cingulat } \\
\text { e Gyrus }\end{array}$} & \multicolumn{3}{|c|}{$\begin{array}{l}\text { Corpus } \\
\text { Callosum }\end{array}$} & \multicolumn{2}{|c|}{$\begin{array}{l}\text { Internal } \\
\text { Capsule }\end{array}$} & \multicolumn{2}{|c|}{$\begin{array}{c}\text { Thalami } \\
\text { c } \\
\text { GM }\end{array}$} & \multicolumn{2}{|c|}{$\begin{array}{l}\text { Brainste } \\
\text { m (Pons) }\end{array}$} & \multicolumn{2}{|c|}{$\begin{array}{c}\text { \% brain regions } \\
\text { stained }\end{array}$} \\
\hline & & & & $\mathrm{Fib}$ & $\lg G$ & $\mathrm{Fib}$ & $\lg C$ & $\mathrm{Fi}$ & & & Fib & $\lg G$ & Fib & $\lg$ & Fib & $\begin{array}{l}\text { Ig } \\
\text { G }\end{array}$ & $\mathrm{Fib}$ & $\lg G$ & Fib & $\lg G$ \\
\hline 1 & 16 & 1 & Moderate (13) & C & C & & & & & & C & C & & & & & & & 83 & 100 \\
\hline 2 & 3 & 5 & Severe (7) & C & C & & & & & & $C$ & C & & & & & & & 71 & 86 \\
\hline 3 & 7 & 3 & Moderate (10) & & & & & & & & $C$ & C & & & & & & & 83 & 83 \\
\hline 4 & 4 & 4 & Severe (8) & & & & & & & & & & & & & & & & 71 & 71 \\
\hline 5 & $<1$ & 2 & Severe (7) & & & & & & & & & & & & & & & & 86 & 100 \\
\hline 6 & 5 & 3 & Severe (3) & & & & & & & & & & & & & & & & 57 & 86 \\
\hline 7 & 13 & 1 & $\begin{array}{l}\text { Moderate } \\
\text { (contusion) }\end{array}$ & & & & & & & & & & & & & & & & 50 & 75 \\
\hline 8 & 8 & 2 & Moderate (11) & & & & & & & & & & & & & & & & 50 & 67 \\
\hline
\end{tabular}




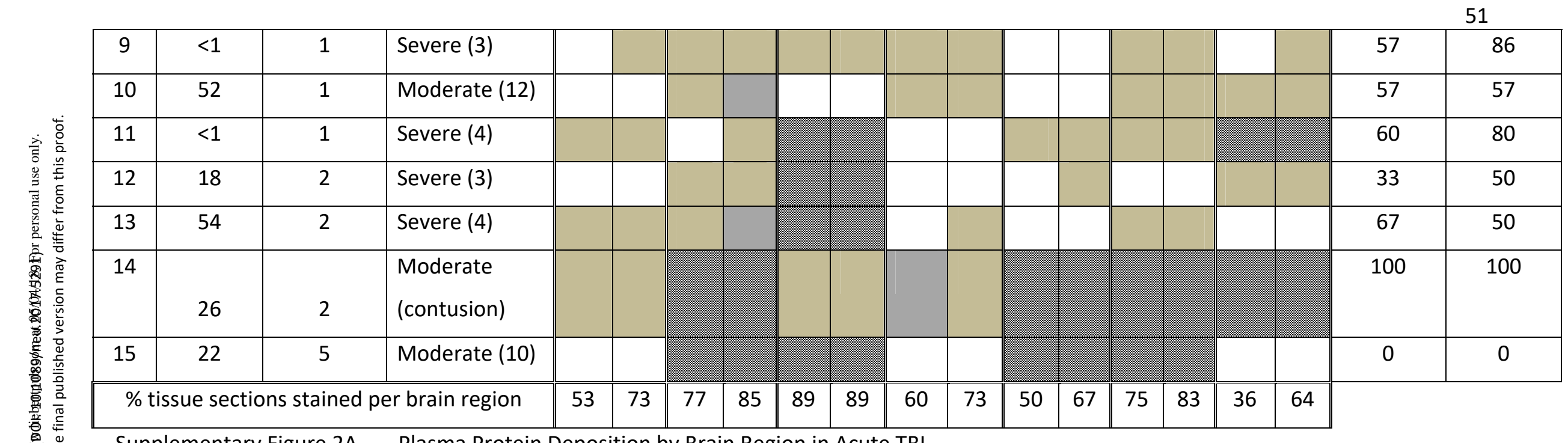

Supplementary Figure 2A Plasma Protein Deposition by Brain Region in Acute TBI 


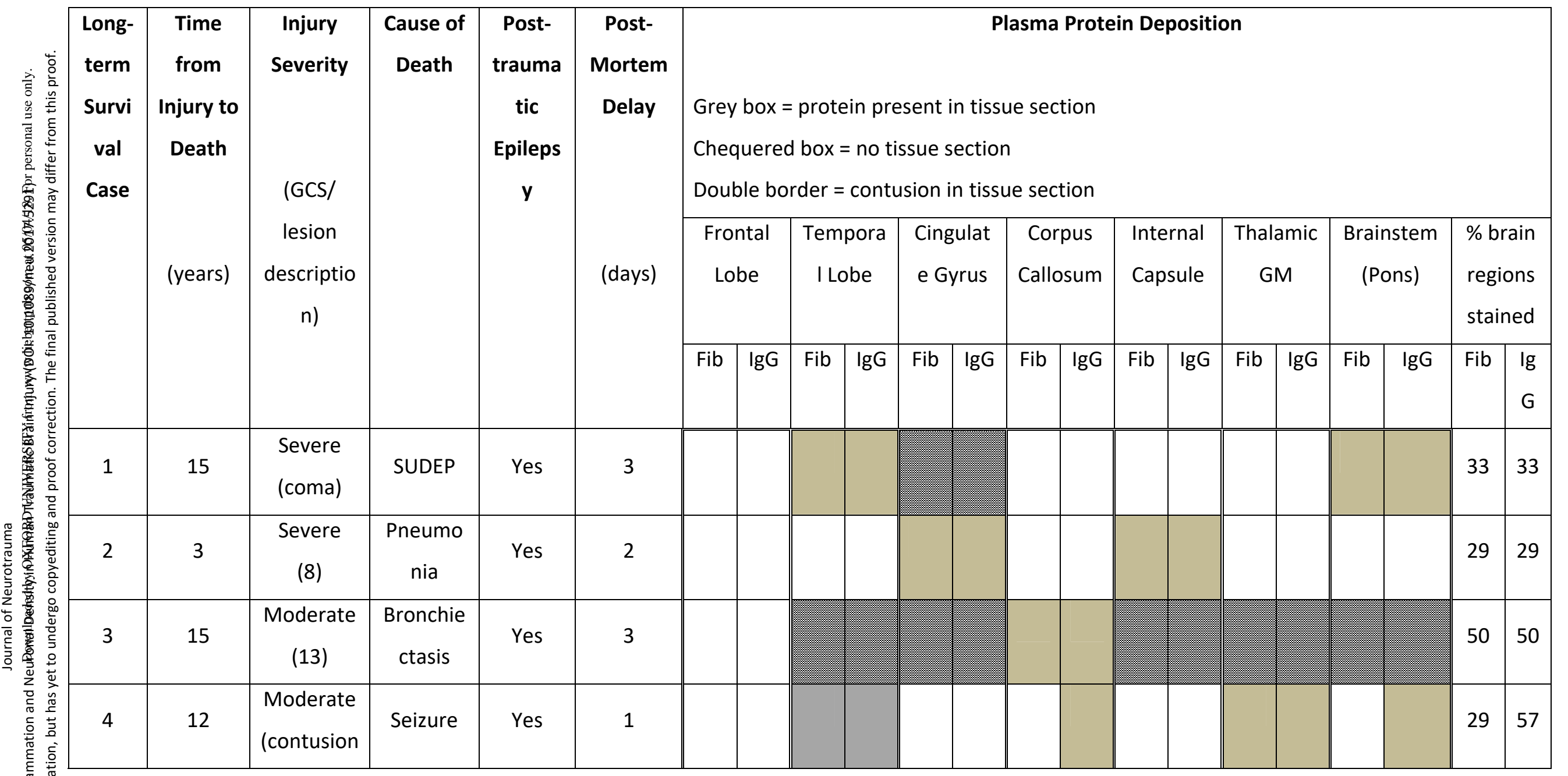




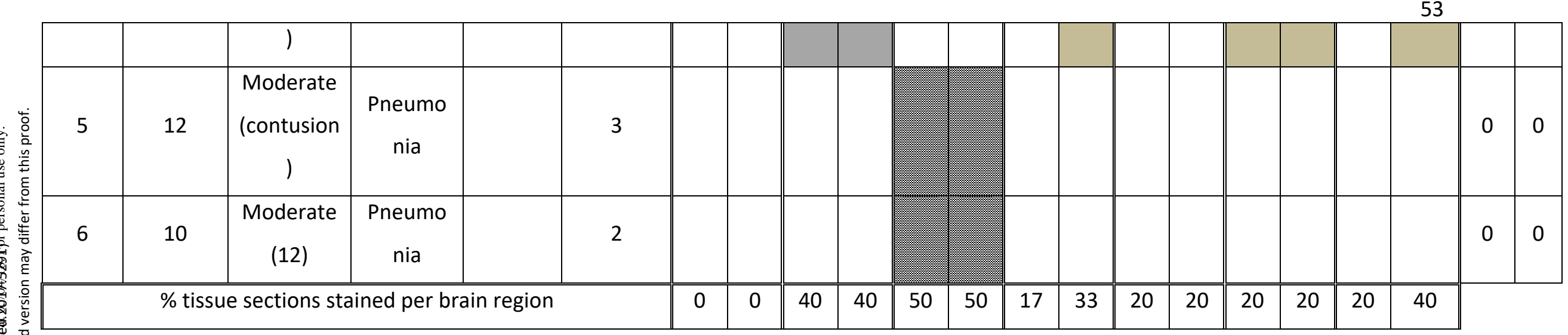

Supplementary Figure 2B Plasma Protein Deposition by Brain Region in Long-Term Survival by Brain Region in Controls 


\begin{tabular}{|c|c|c|c|c|c|c|c|c|c|c|c|c|c|c|c|c|c|c|}
\hline \multirow{4}{*}{$\begin{array}{c}\text { Control } \\
\text { Case }\end{array}$} & \multirow{4}{*}{$\begin{array}{c}\text { Cause of } \\
\text { Death }\end{array}$} & \multirow{4}{*}{$\begin{array}{l}\text { Post- } \\
\text { Mortem } \\
\text { Delay } \\
\text { (days) }\end{array}$} & \\
\hline & & & \multicolumn{16}{|c|}{$\begin{array}{l}\text { 1. Grey box = protein present in tissue section } \\
\text { 2. Chequered box = no tissue section } \\
\text { 3. C = contusion in tissue section }\end{array}$} \\
\hline & & & \multicolumn{2}{|c|}{$\begin{array}{l}\text { Frontal } \\
\text { Lobe }\end{array}$} & \multicolumn{2}{|c|}{$\begin{array}{l}\text { Tempora } \\
\text { I Lobe }\end{array}$} & \multicolumn{2}{|c|}{$\begin{array}{l}\text { Cingulat } \\
\text { e Gyrus }\end{array}$} & \multicolumn{2}{|c|}{$\begin{array}{l}\text { Corpus } \\
\text { Callosum }\end{array}$} & \multicolumn{2}{|c|}{$\begin{array}{l}\text { Internal } \\
\text { Capsule }\end{array}$} & \multicolumn{2}{|c|}{$\begin{array}{c}\text { Thalamic } \\
\text { GM }\end{array}$} & \multicolumn{2}{|c|}{$\begin{array}{l}\text { Brainste } \\
\text { m (Pons) }\end{array}$} & \multicolumn{2}{|c|}{$\begin{array}{l}\text { \% brain } \\
\text { regions } \\
\text { stained }\end{array}$} \\
\hline & & & $\mathrm{Fib}$ & $\lg G$ & Fib & $\lg G$ & Fik & $\operatorname{IgC}$ & $\mathrm{Fib}$ & $\lg C$ & $\mathrm{Fib}$ & $\lg C$ & $\mathrm{Fib}$ & $\lg G$ & Fib & $\lg G$ & $\mathrm{Fib}$ & $\lg G$ \\
\hline 1 & $\begin{array}{l}\text { Myocardial } \\
\text { Infarction }\end{array}$ & 4 & & & & & & & & & & & & & & & 0 & 14 \\
\hline 2 & $\begin{array}{c}\text { Complication } \\
\text { s surgery }\end{array}$ & 2 & & & & & & & & & & & & & & & 0 & 0 \\
\hline 3 & SUDEP & 1 & & & & & & & & & & & & & & & 17 & 17 \\
\hline 4 & $\begin{array}{l}\text { Myocardial } \\
\text { Infarction }\end{array}$ & 2 & & & & & & & & & & & & & & & 0 & 0 \\
\hline 5 & $\begin{array}{c}\text { Oesophageal } \\
\text { cancer }\end{array}$ & 3 & & & & & & & & & & & & & & & 43 & 43 \\
\hline 6 & Oesophageal & 5 & & & & & & & & & & & & & & & 43 & 43 \\
\hline
\end{tabular}




\begin{tabular}{|c|c|c|c|c|c|c|c|c|c|c|c|c|c|c|c|c|c|c|}
\hline & cancer & & & & & & & & & & & & & & & & & \\
\hline 7 & COPD & 1 & & & & & & & & & & & & & & & 60 & 60 \\
\hline 8 & $\begin{array}{c}\text { Complication } \\
\text { s surgery }\end{array}$ & 2 & & & & & & & & & & & & & & & 0 & 14 \\
\hline 9 & COPD & 1 & & & & & & & & & & & & & & & 29 & 29 \\
\hline 10 & $\begin{array}{l}\text { Myocardial } \\
\text { Infarction }\end{array}$ & 1 & & & & & & & & & & & & & & & 43 & 57 \\
\hline 11 & COPD & 3 & & & & & & & & & & & & & & & 67 & 67 \\
\hline 12 & SUDEP & 4 & & & & & & & & & & & & & & & 0 & 0 \\
\hline 13 & SUDEP & 1 & & & & & & & & & & & & & & & 29 & 29 \\
\hline 14 & SUDEP & 2 & & & & & & & & & & & & & & & 33 & 66 \\
\hline 15 & SUDEP & 1 & & & & & & & & & & & & & & & 0 & 17 \\
\hline \multicolumn{3}{|c|}{$\%$ tissue sections stained per brain } & 20 & 27 & 38 & 46 & 15 & 23 & 7 & 20 & 08 & 08 & 31 & 31 & 36 & 46 & & \\
\hline
\end{tabular}

Supplementary Figure 2C Plasma Protein Deposition

Supplementary Figure 2. Plasma protein deposition by brain region in acute TBI (A), in long-term survival (B), and in controls (C). 
Page 56 of 58

\section{Supplemental Figure 3}

Frontal Lobe

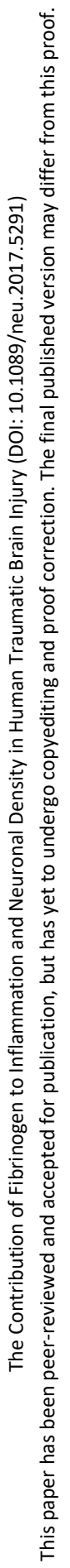

\section{Regional Variation in Fibrinogen Staining}

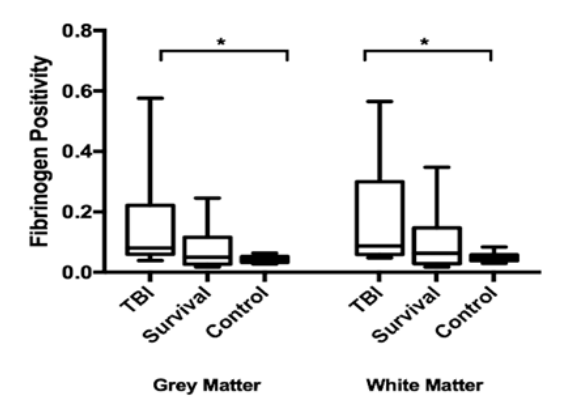

Cingulate Gyrus

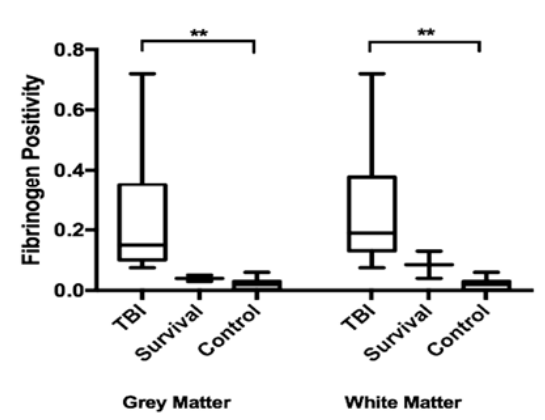

Corpus Callosum \& Brainstem

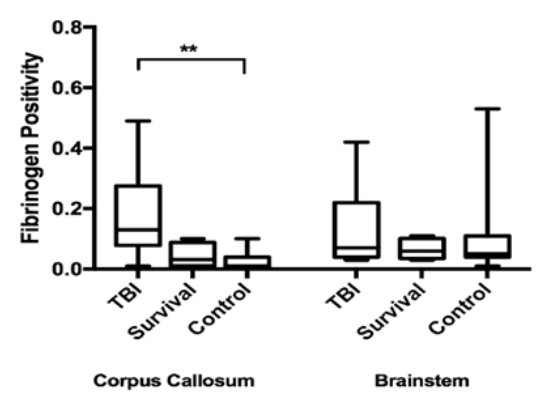

Temporal Lobe

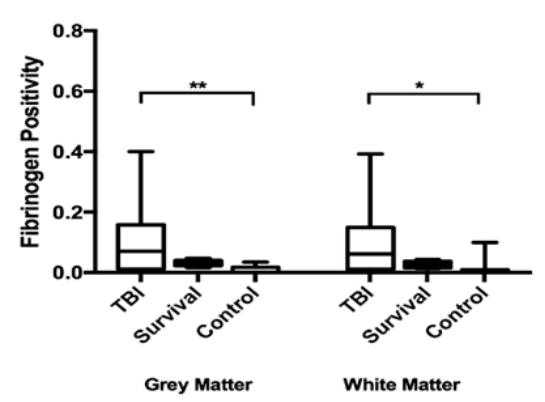

Thalamus \& Internal Capsule

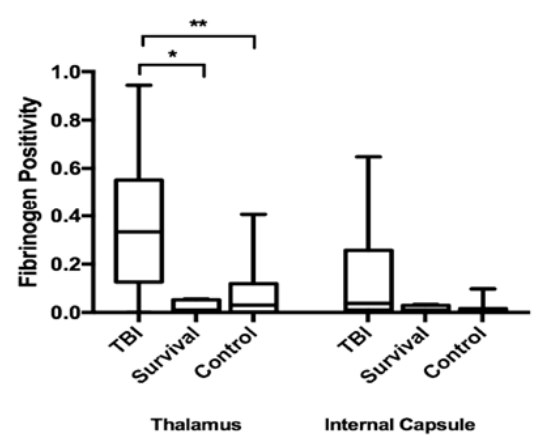

$\underline{\text { Cohorts }}$

$\mathrm{TBI}=$ Acute TBI

Survival $=$ Long-term survival

Control

Supplemental Figure 3. Significant fibrinogen staining in acute TB is found in all brain regions except the internal capsule and brainstem. Here, either wide variation exists in the frequency with which fibrinogen is found (internal capsule) or, alternatively, fibrinogen is also found in cases of long-term sunvival and control (brainstem).

Supplementary Figure 3. Significant fibrinogen staining in acute TBI is found in all brain regions except the internal capsule and brainstem. Here, either wide variation exists in the frequency with which fibrinogen is found (internal capsule) or, alternatively, a similar amount of fibrinogen is seen in cases of acute TBI, long-term survival and control (brainstem). 


\begin{tabular}{|c|c|c|c|}
\hline Brain Region & $\begin{array}{l}\text { Fibrinogen vs } \\
\text { CD68/Iba-1 } \\
\text { Correlation }\end{array}$ & $\begin{array}{c}\text { Fibrinogen vs } \\
\text { Neurons/mm } \\
\text { Correlation }\end{array}$ & $\begin{array}{c}\text { CD68/Iba-1 vs } \\
\text { Neurons/mm } \\
\text { Correlations }\end{array}$ \\
\hline Frontal Lobe & $\begin{array}{l}r_{s} 0.58 \\
C l 0.07-0.84 \\
P 0.027\end{array}$ & $\begin{array}{l}r_{s}-0.72 \\
C l(-0.90)-(-0.32) \\
\text { P } 0.003\end{array}$ & $\begin{array}{l}r_{s}-0.58 \\
C l(-0.85)-(-0.08) \\
P 0.026\end{array}$ \\
\hline Temporal Lobe & $\begin{array}{l}r_{s} 0.72 \\
C l 0.26-0.91 \\
P 0.007\end{array}$ & $\begin{array}{l}r_{s}-0.70 \\
C l(-0.91)-(-0.23) \\
\text { P } 0.009\end{array}$ & $\begin{array}{l}r_{s}-0.85 \\
C l(-0.96)-(-0.55) \\
\text { P } 0.0004\end{array}$ \\
\hline Cingulate Gyrus & $\begin{array}{l}\mathrm{r}_{\mathrm{s}} 0.8 \\
\mathrm{Cl} * \\
\mathrm{P} 0.014\end{array}$ & $\begin{array}{l}\mathrm{r}_{\mathrm{s}}-0.77 \\
\mathrm{Cl} * \\
\mathrm{P} 0.021\end{array}$ & $\begin{array}{l}\mathrm{r}_{\mathrm{s}}-0.80 \\
\mathrm{Cl} * \\
\mathrm{P} 0.014\end{array}$ \\
\hline Thalamus & $\begin{array}{l}r_{s} 0.84 \\
C l 0.53-0.95 \\
P 0.0006\end{array}$ & $\begin{array}{l}r_{s}-0.81 \\
C l(-0.94)-(-0.45) \\
P 0.001\end{array}$ & $\begin{array}{l}r_{s}-0.71 \\
C l(-0.91)-(-0.25) \\
P 0.008\end{array}$ \\
\hline Brainstem & $\begin{array}{l}r_{s} 0.31 \\
C l(-0.34)-0.76 \\
P 0.32\end{array}$ & $\begin{array}{l}r_{s}-0.03 \\
C l(-0.63)-0.59) \\
P 0.93\end{array}$ & $\begin{array}{l}r_{s} 0.08 \\
C l(-0.56)-0.66 \\
P 0.82\end{array}$ \\
\hline
\end{tabular}

Supplementary Figure 4. Regional grey matter correlations: fibrinogen, CD68/Iba-1, neurons $/ \mathrm{mm}^{2}$. 
Page 58 of 58

Supplementary Figure 5

A

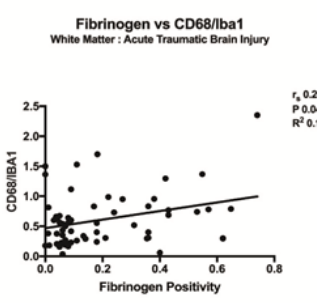

FIBRINOGEN

B
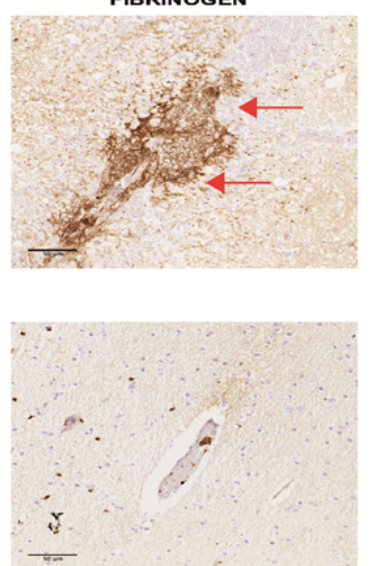

Supplementary Figure 5. Correlations between white matter fibrinogen, CD68/lba-1 and $\beta A P P$ in acute TBI (A). Although no overall correlation emerged between fibrinogen deposition and $\beta A P P$ staining, fibrinogen was, rarely, noted to cuff blood vessels. This created the appearance of a web of parenchymal fibrin/fibrinogen (red arrow). Spatially overlapping the fibrinogen were activated microglia/macrophages, denoted by perivascular CD68 accumulation, and BAPP-stained axonal spheroids (B, upper row, spheroids marked by black arrows). Blood vessels in the same tissue section that lacked perivascular fibrinogen were devoid of inflammation or axonal spheroids (B, lower row).

Supplementary Figure 5. (A) Correlations between WM fibrinogen, CD68/Iba-1, and $\beta$ APP in acute TBI. (B) Although no overall correlation emerged between fibrinogen deposition and $\beta$ APP staining, fibrinogen was, rarely, noted to cuff blood vessels (BV). This created the appearance of a web of parenchymal fibrin/fibrinogen (red arrow). Spatially overlapping the fibrinogen web were activated microglia/macrophages (denoted by perivascular CD68 accumulation) and $\beta$ APP-stained axonal spheroids (upper row, black arrows). Blood vessels in the same tissue section that lacked perivascular fibrinogen were devoid of inflammation and axonal spheroids (lower row). 